\title{
MECHANISM AND KINETIC MODEL OF CHLORATE AND PERCHLORATE FORMATION DURING OZONATION OF AQUEOUS CHLORIDE SOLUTIONS
}

\author{
Alexander V. Levanov, ${ }^{*}$ Oksana Ya. Isaikina \\ Department of Chemistry, M.V. Lomonosov Moscow State University, Leninskiye \\ Gory 1, building 3, 119991 Moscow (Russia) \\ * Corresponding Author. \\ E-mail address: levanov@kge.msu.ru \\ Fax: (+7) 495-939-4575; Phone: (+7) 495-939-3685
}


This Supporting Information contains:

INITIAL SET OF CHEMICAL REACTIONS IN THE SYSTEM O ${ }_{3}-\mathrm{Cl}^{-}$IN

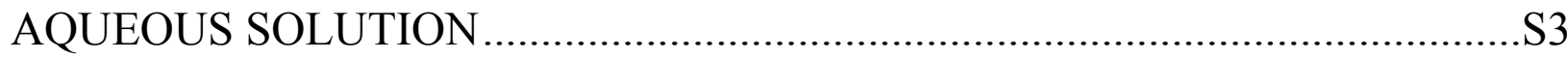

EXAMPLES OF KINETIC CURVES CALCULATED WITH THE MODEL.....S9 REDUCED SET OF CHEMICAL REACTIONS IN THE SYSTEM O ${ }_{3}-\mathrm{Cl}^{-}$IN

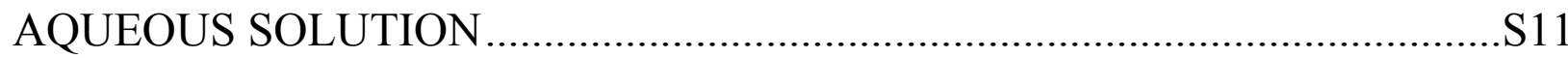

THE EFFECT OF UNCERTAINTY IN THE RATE CONSTANT OF REACTION $\mathrm{O}_{3}+\mathrm{OH}^{-} \rightarrow \mathrm{HO}_{2}{ }^{-}+\mathrm{O}_{2}$ ON THE FITTED VALUE OF THE RATE CONSTANT OF $\mathrm{Cl}_{2}{ }^{-}+\mathrm{O}_{3} \rightarrow \mathrm{ClO}^{*}+\mathrm{Cl}^{-}+\mathrm{O}_{2}$ REACTION ...........................S14

KINETIC ORDER OF CHLORATE FORMATION REACTION WITH RESPECT TO OZONE AND CHLORIDE ..........................................................S16

EXPERIMENTAL AND CALCULATED RATES OF PERCHLORATE FORMATION ON OZONATION OF CHLORIDE SOLUTIONS .....................S18 ESTIMATION OF STANDARD GIBBS ENERGY OF REACTION $\mathrm{ClO}_{3} \cdot+\mathrm{O}_{3}$ $\rightarrow \mathrm{ClO}_{2} \cdot+2 \mathrm{O}_{2}$ IN AQUEOUS SOLUTION S19

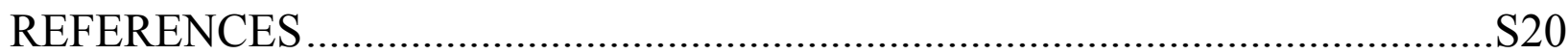




\section{INITIAL SET OF CHEMICAL REACTIONS IN THE SYSTEM $\mathrm{O}_{3}-\mathrm{Cl}^{-}$IN AQUEOUS SOLUTION}

The units of rate constants are $\mathrm{s}^{-1}$ for first order reactions, and $\mathrm{L} \mathrm{mol}^{-1} \mathrm{~s}^{-1}$ for second order reactions. Equilibrium constants are expressed in mol/L units. The equilibrium constants for the process of gas dissolution, $\mathrm{A}$ (gas) $\rightleftarrows \mathrm{A}(\mathrm{aq})$, are true Henry's law constants, $H_{\mathrm{A}}$.

Whenever possible, the rate and equilibrium constants are given for temperature 20 ${ }^{\circ} \mathrm{C}$ and ionic strength $1 \mathrm{M}$. Otherwise, the constants corresponds to $25^{\circ} \mathrm{C}$ and the conditions specified in the cited publication.

Table S1. Equilibrium Constants of Reversible Reactions

\begin{tabular}{|c|c|c|c|}
\hline No & Process & Equilibrium Constant & Ref \\
\hline 1 & $\mathrm{O}_{2}($ gas $) \rightleftarrows \mathrm{O}_{2}(\mathrm{aq})$ & 0.035 & {$[1]$} \\
\hline 2 & $\mathrm{O}_{3}($ gas $) \rightleftarrows \mathrm{O}_{3}(\mathrm{aq})$ & 0.18 & [2] \\
\hline 3 & $\mathrm{Cl}_{2}($ gas $) \rightleftarrows \mathrm{Cl}_{2}(\mathrm{aq})$ & 1.51 & [3] \\
\hline 4 & $\mathrm{HOCl}(\mathrm{gas}) \rightleftarrows \mathrm{HOCl}(\mathrm{aq})$ & $2.2 \times 10^{5}$ & [4] \\
\hline 5 & $\mathrm{ClO}(\mathrm{gas}) \rightleftarrows \mathrm{ClO}(\mathrm{aq})$ & $2.0 \times 10^{4}$ & [5] \\
\hline 6 & $\mathrm{ClO}_{2}($ gas $) \rightleftarrows \mathrm{ClO}_{2}(\mathrm{aq})$ & 24 & [6] \\
\hline 7 & $\mathrm{H}_{2} \mathrm{O} \rightleftarrows \mathrm{H}^{+}+\mathrm{OH}^{-}$ & $0.67 \times 10^{-14}$ & [7] \\
\hline 8 & $\mathrm{H}_{2} \mathrm{O}_{2} \rightleftarrows \mathrm{H}^{+}+\mathrm{HO}_{2}^{-}$ & $1.44 \times 10^{-12}$ & [7] \\
\hline 9 & $\mathrm{OH}+\mathrm{OH}^{-} \rightleftarrows \mathrm{O}^{-}+\mathrm{H}_{2} \mathrm{O}$ & 305 & [8] \\
\hline 10 & $\mathrm{HO}_{2} \rightleftarrows \mathrm{H}^{+}+\mathrm{O}_{2}^{-}$ & $1.46 \times 10^{-5}$ & [7] \\
\hline 11 & $\mathrm{HO}_{3} \rightleftarrows \mathrm{H}^{+}+\mathrm{O}_{3}^{-}$ & $6.31 \times 10^{-9}$ & {$[9,10]$} \\
\hline 12 & $\mathrm{HOCl} \rightleftarrows \mathrm{H}^{+}+\mathrm{ClO}^{-}$ & $3.92 \times 10^{-8}$ & {$[11,12]$} \\
\hline 13 & $\mathrm{HClO}_{2} \rightleftarrows \mathrm{H}^{+}+\mathrm{ClO}_{2}^{-}$ & $1.91 \times 10^{-2}$ & {$[13]$} \\
\hline 14 & $\mathrm{Cl}_{2}+\mathrm{H}_{2} \mathrm{O} \rightleftarrows \mathrm{H}^{+}+\mathrm{Cl}^{-}+\mathrm{HOCl}$ & $7.50 \times 10^{-4}$ & {$[14]$} \\
\hline 15 & $\mathrm{Cl}^{-}+\mathrm{Cl}_{2} \rightleftarrows \mathrm{Cl}_{3}^{-}$ & 0.18 & {$[15,16]$} \\
\hline
\end{tabular}


Table S2. Reactions and Rate Constants

\begin{tabular}{|c|c|c|c|}
\hline No. & Reaction & Rate Constant & Ref \\
\hline 1 & $\mathrm{O}_{2}($ gas $) \rightarrow 0.055 \mathrm{O}_{2}$ & 0.0063 & {$[1]$} \\
\hline 2 & $\mathrm{O}_{2} \rightarrow 18.2 \mathrm{O}_{2}$ (gas) & 0.1 & {$[17]$} \\
\hline 3 & $\mathrm{O}_{3}($ gas $) \rightarrow 0.055 \mathrm{O}_{3}$ & 0.33 & {$[2]$} \\
\hline 4 & $\mathrm{O}_{3} \rightarrow 18.2 \mathrm{O}_{3}$ (gas) & 0.1 & {$[17]$} \\
\hline 5 & $\mathrm{Cl}_{2}$ (gas) $\rightarrow 0.055 \mathrm{Cl}_{2}$ & 2.8 & {$[3]$} \\
\hline 6 & $\mathrm{Cl}_{2} \rightarrow 18.2 \mathrm{Cl}_{2}$ (gas) & 0.1 & [17] \\
\hline 7 & $\mathrm{HOCl}($ gas $) \rightarrow 0.055 \mathrm{HOCl}$ & $4.1 \times 10^{4}$ & [4] \\
\hline 8 & $\mathrm{HOCl} \rightarrow 18.2 \mathrm{HOCl}$ (gas) & 0.1 & {$[17]$} \\
\hline 9 & $\mathrm{ClO}($ gas $) \rightarrow 0.055 \mathrm{ClO}$ & $3.6 \times 10^{4}$ & [5] \\
\hline 10 & $\mathrm{ClO} \rightarrow 18.2 \mathrm{ClO}$ (gas) & 0.1 & [17] \\
\hline 11 & $\mathrm{ClO}_{2}$ (gas) $\rightarrow 0.055 \mathrm{ClO}_{2}$ & 44 & [6] \\
\hline 12 & $\mathrm{ClO}_{2} \rightarrow 18.2 \mathrm{ClO}_{2}$ (gas) & 0.1 & {$[17]$} \\
\hline 13 & $\mathrm{H}_{2} \mathrm{O} \rightarrow \mathrm{H}^{+}+\mathrm{OH}^{-}$ & $6.72 \times 10^{-4}$ & [7] \\
\hline 14 & $\mathrm{H}^{+}+\mathrm{OH}^{-} \rightarrow \mathrm{H}_{2} \mathrm{O}$ & $1.00 \times 10^{11}$ & [7] \\
\hline 15 & $\mathrm{H}_{2} \mathrm{O}_{2} \rightarrow \mathrm{H}^{+}+\mathrm{HO}_{2}^{-}$ & $7.18 \times 10^{-2}$ & [7] \\
\hline 16 & $\mathrm{H}^{+}+\mathrm{HO}_{2}^{-} \rightarrow \mathrm{H}_{2} \mathrm{O}_{2}$ & $5.00 \times 10^{10}$ & \\
\hline 17 & $\mathrm{OH}+\mathrm{OH}^{-} \rightarrow \mathrm{O}^{-}+\mathrm{H}_{2} \mathrm{O}$ & $1.17 \times 10^{10}$ & [7] \\
\hline 18 & $\mathrm{O}^{-}\left(+\mathrm{H}_{2} \mathrm{O}\right) \rightarrow \mathrm{OH}+\mathrm{OH}^{-}$ & $3.84 \times 10^{7}(\mathrm{~A})$ & [8] \\
\hline 19 & $\mathrm{HO}_{2} \rightarrow \mathrm{H}^{+}+\mathrm{O}_{2}^{-}$ & $6.62 \times 10^{5}$ & [7] \\
\hline 20 & $\mathrm{H}^{+}+\mathrm{O}_{2}^{-} \rightarrow \mathrm{HO}_{2}$ & $4.52 \times 10^{10}$ & [7] \\
\hline 21 & $\mathrm{HO}_{3} \rightarrow \mathrm{H}^{+}+\mathrm{O}_{3}^{-}$ & $3.28 \times 10^{2}$ & {$[9,10]$} \\
\hline 22 & $\mathrm{H}^{+}+\mathrm{O}_{3}^{-} \rightarrow \mathrm{HO}_{3}$ & $5.20 \times 10^{10}$ & [9] \\
\hline 23 & $\mathrm{HO}_{3} \rightarrow \mathrm{OH}+\mathrm{O}_{2}$ & $1.10 \times 10^{5}$ & [9] \\
\hline 24 & $\mathrm{HOCl} \rightarrow \mathrm{H}^{+}+\mathrm{ClO}^{-}$ & $2.0 \times 10^{3}$ & {$[11,12]$} \\
\hline 25 & $\mathrm{H}^{+}+\mathrm{ClO}^{-} \rightarrow \mathrm{HOCl}$ & $5 \times 10^{10}$ & \\
\hline 26 & $\mathrm{HClO}_{2} \rightarrow \mathrm{H}^{+}+\mathrm{ClO}_{2}^{-}$ & $9.53 \times 10^{8}$ & [13] \\
\hline 27 & $\mathrm{H}^{+}+\mathrm{ClO}_{2}^{-} \rightarrow \mathrm{HClO}_{2}$ & $5 \times 10^{10}$ & \\
\hline 28 & $\mathrm{Cl}_{2}\left(+\mathrm{H}_{2} \mathrm{O}\right) \rightarrow \mathrm{H}^{+}+\mathrm{Cl}^{-}+\mathrm{HOCl}$ & $22(\mathrm{~A})$ & {$[14]$} \\
\hline 29 & $\mathrm{Cl}_{2}+\mathrm{OH}^{-} \rightarrow \mathrm{Cl}^{-}+\mathrm{HOCl}$ & $6.00 \times 10^{8}$ & {$[18]$} \\
\hline 30 & $\mathrm{H}^{+}+\mathrm{Cl}^{-}+\mathrm{HOCl} \rightarrow \mathrm{Cl}_{2}+\mathrm{H}_{2} \mathrm{O}$ & $2.10 \times 10^{4}$ & {$[14]$} \\
\hline 31 & $\mathrm{Cl}_{3}^{-} \rightarrow \mathrm{Cl}^{-}+\mathrm{Cl}_{2}$ & $1.11 \times 10^{5}$ & {$[15]$} \\
\hline
\end{tabular}




\begin{tabular}{|c|c|c|c|}
\hline 32 & $\mathrm{Cl}^{-}+\mathrm{Cl}_{2} \rightarrow \mathrm{Cl}_{3}^{-}$ & $2.00 \times 10^{4}$ & [15] \\
\hline 33 & $\mathrm{O}_{3}+\mathrm{Cl}^{-} \rightarrow \mathrm{ClO}^{-}+\mathrm{O}_{2}$ & $\left({ }^{\mathrm{B}}\right)$ & [2] \\
\hline 34 & $\mathrm{ClO}^{-}+\mathrm{O}_{3} \rightarrow \mathrm{Cl}^{-}+2 \mathrm{O}_{2}$ & $73(\mathrm{C})$ & [19] \\
\hline 35 & $\mathrm{ClO}^{-}+\mathrm{O}_{3} \rightarrow \mathrm{ClO}_{2}^{-}+\mathrm{O}_{2}$ & $21(\mathrm{C})$ & [19] \\
\hline 36 & $\mathrm{ClO}_{2}^{-}+\mathrm{O}_{3} \rightarrow \mathrm{ClO}_{2}+\mathrm{O}_{3}^{-}$ & $8.20 \times 10^{6}$ & {$[20,21]$} \\
\hline 37 & $\mathrm{ClO}_{2}^{-}+\mathrm{OH} \rightarrow \mathrm{ClO}_{2}+\mathrm{OH}^{-}$ & $8.00 \times 10^{9}$ & [22] \\
\hline 38 & $\mathrm{ClO}_{2}^{-}+\mathrm{O}^{-} \rightarrow \mathrm{ClO}_{2}+\mathrm{OH}^{-}$ & $2.00 \times 10^{8}$ & [23] \\
\hline 39 & $\mathrm{ClO}_{2}+\mathrm{O}_{3} \rightarrow \mathrm{ClO}_{3}+\mathrm{O}_{2}$ & $1.40 \times 10^{3}$ & [24] \\
\hline 40 & $\mathrm{ClO}_{2}+\mathrm{O}_{3}^{-} \rightarrow \mathrm{ClO}_{2}^{-}+\mathrm{O}_{3}$ & $1.80 \times 10^{5}$ & {$[20,25]$} \\
\hline 41 & $\mathrm{ClO}_{2}+\mathrm{O}_{3}^{-} \rightarrow \mathrm{ClO}_{3}^{-}+\mathrm{O}_{2}$ & $1.80 \times 10^{5}$ & [20] \\
\hline 42 & $\mathrm{ClO}_{2}+\mathrm{OH} \rightarrow \mathrm{H}^{+}+\mathrm{ClO}_{3}^{-}$ & $2.60 \times 10^{9}$ & [26] \\
\hline 43 & $\mathrm{ClO}_{2}+\mathrm{OH} \rightarrow \mathrm{HOCl}+\mathrm{O}_{2}$ & $1.40 \times 10^{9}$ & [26] \\
\hline 44 & $\mathrm{ClO}_{2}+\mathrm{O}^{-} \rightarrow \mathrm{ClO}_{3}^{-}$ & $2.20 \times 10^{9}$ & [26] \\
\hline 45 & $\mathrm{ClO}_{2}+\mathrm{O}^{-} \rightarrow \mathrm{ClO}^{-}+\mathrm{O}_{2}$ & $4.90 \times 10^{8}$ & [26] \\
\hline 46 & $\mathrm{Cl}^{-}+\mathrm{OH} \rightarrow \mathrm{ClOH}^{-}$ & $4.20 \times 10^{9}$ & [27] \\
\hline 47 & $\mathrm{ClOH}^{-} \rightarrow \mathrm{Cl}^{-}+\mathrm{OH}$ & $6.10 \times 10^{9}$ & [27] \\
\hline 48 & $\mathrm{ClOH}^{-} \rightarrow \mathrm{Cl}+\mathrm{OH}^{-}$ & 23 & {$[28,29]$} \\
\hline 49 & $\mathrm{Cl}+\mathrm{OH}^{-} \rightarrow \mathrm{ClOH}^{-}$ & $1.80 \times 10^{10}$ & {$[28,29]$} \\
\hline 50 & $\mathrm{ClOH}^{-}+\mathrm{H}^{+} \rightarrow \mathrm{Cl}+\mathrm{H}_{2} \mathrm{O}$ & $2.40 \times 10^{10}$ & [27] \\
\hline 51 & $\mathrm{Cl}\left(+\mathrm{H}_{2} \mathrm{O}\right) \rightarrow \mathrm{ClOH}^{-}+\mathrm{H}^{+}$ & $1.80 \times 10^{5}\left({ }^{\mathrm{A}}\right)$ & [27] \\
\hline 52 & $\mathrm{Cl}+\mathrm{Cl}^{-} \rightarrow \mathrm{Cl}_{2}^{-}$ & $7.80 \times 10^{9}$ & [27] \\
\hline 53 & $\mathrm{Cl}_{2}^{-} \rightarrow \mathrm{Cl}+\mathrm{Cl}^{-}$ & $5.70 \times 10^{4}$ & [27] \\
\hline 54 & $\mathrm{Cl}_{2}^{-}\left(+\mathrm{H}_{2} \mathrm{O}\right) \rightarrow \mathrm{ClOH}^{-}+\mathrm{H}^{+}+\mathrm{Cl}^{-}$ & $1.30 \times 10^{3}\left({ }^{\mathrm{A}}\right)$ & [27] \\
\hline 55 & $\mathrm{Cl}^{-}+\mathrm{ClOH}^{-} \rightarrow \mathrm{Cl}_{2}^{-}+\mathrm{OH}^{-}$ & $1.00 \times 10^{4}$ & [30] \\
\hline 56 & $\mathrm{Cl}_{2}^{-}+\mathrm{OH}^{-} \rightarrow \mathrm{Cl}^{-}+\mathrm{ClOH}^{-}$ & $4.50 \times 10^{7}$ & [30] \\
\hline 57 & $\mathrm{Cl}+\mathrm{Cl} \rightarrow \mathrm{Cl}_{2}$ & $8.80 \times 10^{7}$ & [31] \\
\hline 58 & $\mathrm{Cl}+\mathrm{Cl}_{2}^{-} \rightarrow \mathrm{Cl}_{2}+\mathrm{Cl}^{-}$ & $1.40 \times 10^{9}$ & [27] \\
\hline 59 & $\mathrm{Cl}_{2}^{-}+\mathrm{Cl}_{2}^{-} \rightarrow \mathrm{Cl}_{2}+2 \mathrm{Cl}^{-}$ & $3.50 \times 10^{9}$ & [27] \\
\hline 60 & $\mathrm{Cl}_{2}^{-}+\mathrm{OH} \rightarrow \mathrm{HOCl}+\mathrm{Cl}^{-}$ & $1.00 \times 10^{9}$ & [32] \\
\hline 61 & $\mathrm{Cl}_{2}^{-}+\mathrm{O}_{3} \rightarrow \mathrm{ClO}+\mathrm{Cl}^{-}+\mathrm{O}_{2}$ & $9.00 \times 10^{7}\left({ }^{\mathrm{C}}\right)$ & [33] \\
\hline 62 & $\mathrm{HOCl}+\mathrm{Cl} \rightarrow \mathrm{ClO}+\mathrm{Cl}^{-}+\mathrm{H}^{+}$ & $3.00 \times 10^{9}$ & [28] \\
\hline 63 & $\mathrm{ClO}^{-}+\mathrm{Cl} \rightarrow \mathrm{ClO}+\mathrm{Cl}^{-}$ & $8.20 \times 10^{9}$ & [28] \\
\hline 64 & $\mathrm{HOCl}+\mathrm{OH} \rightarrow \mathrm{ClO}$ & $1.20 \times 10^{8}$ & [22] \\
\hline 65 & $\mathrm{ClO}^{-}+\mathrm{OH} \rightarrow \mathrm{ClO}+\mathrm{OH}^{-}$ & $3.00 \times 10^{9}$ & {$[22]$} \\
\hline
\end{tabular}




\begin{tabular}{|c|c|c|c|}
\hline 66 & $\mathrm{ClO}^{-}+\mathrm{O}^{-} \rightarrow \mathrm{ClO}+\mathrm{OH}^{-}$ & $2.30 \times 10^{8}$ & [25] \\
\hline 67 & $\mathrm{ClO}+\mathrm{ClO}_{2}^{-} \rightarrow \mathrm{ClO}^{-}+\mathrm{ClO}_{2}$ & $9.40 \times 10^{8}$ & [34] \\
\hline 68 & $\mathrm{Cl}+\mathrm{ClO}_{2} \rightarrow \mathrm{Cl}_{2} \mathrm{O}_{2}$ & $1.00 \times 10^{9}$ & [35] \\
\hline 69 & $\mathrm{Cl}_{2}^{-}+\mathrm{ClO}_{2} \rightarrow \mathrm{Cl}_{2} \mathrm{O}_{2}+\mathrm{Cl}^{-}$ & $1.00 \times 10^{9}$ & [35] \\
\hline 70 & $\mathrm{ClO}+\mathrm{O}_{3}^{-} \rightarrow \mathrm{ClO}^{-}+\mathrm{O}_{3}$ & $1.00 \times 10^{9}$ & [36] \\
\hline 71 & $\mathrm{Cl}+\mathrm{O}_{3}^{-} \rightarrow \mathrm{Cl}^{-}+\mathrm{O}_{3}$ & $1.00 \times 10^{9}$ & [36] \\
\hline 72 & $\mathrm{Cl}^{-}+\mathrm{H}_{2} \mathrm{O}_{2} \rightarrow \mathrm{ClO}^{-}+\mathrm{H}_{2} \mathrm{O}$ & $1.80 \times 10^{-9}$ & [37] \\
\hline 73 & $\mathrm{Cl}^{-}+\mathrm{H}_{2} \mathrm{O}_{2}+\mathrm{H}^{+} \rightarrow \mathrm{HOCl}+\mathrm{H}_{2} \mathrm{O}$ & $8.30 \times 10^{-7}$ & [37] \\
\hline 74 & $\mathrm{ClO}^{-}+\mathrm{H}_{2} \mathrm{O}_{2} \rightarrow \mathrm{Cl}^{-}+\mathrm{O}_{2}+\mathrm{H}_{2} \mathrm{O}$ & $3.40 \times 10^{3}$ & [38] \\
\hline 75 & $\mathrm{HOCl}+\mathrm{HO}_{2}^{-} \rightarrow \mathrm{Cl}^{-}+\mathrm{O}_{2}+\mathrm{H}_{2} \mathrm{O}$ & $4.40 \times 10^{7}$ & [38] \\
\hline 76 & $\mathrm{Cl}_{2}+\mathrm{H}_{2} \mathrm{O}_{2} \rightarrow 2 \mathrm{Cl}^{-}+\mathrm{O}_{2}+2 \mathrm{H}^{+}$ & $\left({ }^{\mathrm{D}}\right)$ & [38-40] \\
\hline 77 & $\mathrm{Cl}_{2}+\mathrm{HO}_{2}^{-} \rightarrow 2 \mathrm{Cl}^{-}+\mathrm{O}_{2}+\mathrm{H}^{+}$ & $1.10 \times 10^{8}$ & {$[41,42]$} \\
\hline 78 & $\mathrm{Cl}_{2}+\mathrm{HO}_{2} \rightarrow \mathrm{Cl}_{2}^{-}+\mathrm{H}^{+}+\mathrm{O}_{2}$ & $1.00 \times 10^{9}$ & [43] \\
\hline 79 & $\mathrm{HOCl}+\mathrm{O}_{2}^{-} \rightarrow \mathrm{Cl}^{-}+\mathrm{O}_{2}+\mathrm{OH}$ & $7.50 \times 10^{6}$ & [44] \\
\hline 80 & $\mathrm{Cl}+\mathrm{H}_{2} \mathrm{O}_{2} \rightarrow \mathrm{Cl}^{-}+\mathrm{H}^{+}+\mathrm{HO}_{2}$ & $2.00 \times 10^{9}$ & [27] \\
\hline 81 & $\mathrm{Cl}+\mathrm{HO}_{2} \rightarrow \mathrm{Cl}^{-}+\mathrm{H}^{+}+\mathrm{O}_{2}$ & $3.10 \times 10^{9}$ & [45] \\
\hline 82 & $\mathrm{Cl}_{2}^{-}+\mathrm{H}_{2} \mathrm{O}_{2} \rightarrow 2 \mathrm{Cl}^{-}+\mathrm{H}^{+}+\mathrm{HO}_{2}$ & $6.20 \times 10^{6}$ & [27] \\
\hline 83 & $\mathrm{Cl}_{2}^{-}+\mathrm{HO}_{2} \rightarrow 2 \mathrm{Cl}^{-}+\mathrm{H}^{+}+\mathrm{O}_{2}$ & $3.10 \times 10^{9}$ & [27] \\
\hline 84 & $\mathrm{Cl}_{2}^{-}+\mathrm{O}_{2}^{-} \rightarrow 2 \mathrm{Cl}^{-}+\mathrm{O}_{2}$ & $2.00 \times 10^{9}$ & [46] \\
\hline 85 & $\mathrm{ClO}_{2}+\mathrm{H}_{2} \mathrm{O}_{2} \rightarrow \mathrm{ClO}_{2}^{-}+\mathrm{H}^{+}+\mathrm{HO}_{2}$ & 4.0 & [23] \\
\hline 86 & $\mathrm{ClO}_{2}+\mathrm{HO}_{2}^{-} \rightarrow \mathrm{ClO}_{2}^{-}+\mathrm{HO}_{2}$ & $1.30 \times 10^{5}$ & [24] \\
\hline 87 & $\mathrm{ClO}_{2}+\mathrm{HO}_{2} \rightarrow \mathrm{ClO}_{2}^{-}+\mathrm{H}^{+}+\mathrm{O}_{2}$ & $1.00 \times 10^{6}$ & [47] \\
\hline 88 & $\mathrm{ClO}_{2}+\mathrm{O}_{2}^{-} \rightarrow \mathrm{ClO}_{2}^{-}+\mathrm{O}_{2}$ & $3.00 \times 10^{9}$ & {$[23,47]$} \\
\hline 89 & $\mathrm{ClO}_{2}^{-}+\mathrm{O}_{2}^{-} \rightarrow \mathrm{ClO}^{-}+\mathrm{O}_{2}+\mathrm{O}^{-}$ & 40 & [44] \\
\hline 90 & $\mathrm{ClO}+\mathrm{ClO} \rightarrow \mathrm{Cl}_{2} \mathrm{O}_{2}$ & $2.50 \times 10^{9}$ & [28] \\
\hline 91 & $\mathrm{ClO}+\mathrm{ClO}_{2} \rightarrow \mathrm{ClOClO}_{2}$ & $7.40 \times 10^{9}$ & [48] \\
\hline 92 & $\mathrm{ClO}+\mathrm{ClO}_{3} \rightarrow \mathrm{ClOClO}_{3}$ & $7.40 \times 10^{9}$ & [48] \\
\hline 93 & $\mathrm{ClO}_{2}+\mathrm{ClO}_{3} \rightarrow \mathrm{O}_{2} \mathrm{ClOClO}_{2}$ & $7.40 \times 10^{9}$ & [48] \\
\hline 94 & $\mathrm{ClO}_{3}+\mathrm{ClO}_{3} \rightarrow \mathrm{O}_{2} \mathrm{ClOClO}_{3}$ & $7.40 \times 10^{9}$ & [48] \\
\hline 95 & $\mathrm{Cl}_{2} \mathrm{O}_{2}\left(+\mathrm{H}_{2} \mathrm{O}\right) \rightarrow \mathrm{Cl}^{-}+\mathrm{ClO}_{3}^{-}+2 \mathrm{H}^{+}$ & $1.00 \times 10^{4}\left({ }^{\mathrm{A}}\right)$ & [48] \\
\hline 96 & $\mathrm{Cl}_{2} \mathrm{O}_{2}+\mathrm{OH}^{-} \rightarrow \mathrm{Cl}^{-}+\mathrm{ClO}_{3}^{-}+\mathrm{H}^{+}$ & $1.00 \times 10^{10}$ & [48] \\
\hline 97 & $\mathrm{ClOClO}_{2}\left(+\mathrm{H}_{2} \mathrm{O}\right) \rightarrow \mathrm{HOCl}+\mathrm{ClO}_{3}^{-}+\mathrm{H}^{+}$ & $1.00 \times 10^{4}\left({ }^{\mathrm{A}}\right)$ & [48] \\
\hline 98 & $\mathrm{ClOClO}_{3}\left(+\mathrm{H}_{2} \mathrm{O}\right) \rightarrow \mathrm{HOCl}+\mathrm{ClO}_{4}^{-}+\mathrm{H}^{+}$ & $1.00 \times 10^{4}\left({ }^{\mathrm{A}}\right)$ & [48] \\
\hline 99 & $\mathrm{O}_{2} \mathrm{ClOClO}_{2}\left(+\mathrm{H}_{2} \mathrm{O}\right) \rightarrow 2 \mathrm{ClO}_{3}^{-}+2 \mathrm{H}^{+}$ & $1.00 \times 10^{4}\left({ }^{\mathrm{A}}\right)$ & [48] \\
\hline
\end{tabular}




\begin{tabular}{|c|c|c|c|}
\hline 100 & $\mathrm{O}_{2} \mathrm{ClOClO}_{3}\left(+\mathrm{H}_{2} \mathrm{O}\right) \rightarrow \mathrm{ClO}_{3}^{-}+\mathrm{ClO}_{4}^{-}+2 \mathrm{H}^{+}$ & $1.00 \times 10^{4}\left({ }^{\mathrm{A}}\right)$ & [48] \\
\hline 101 & $\mathrm{O}_{3}+\mathrm{OH}^{-} \rightarrow \mathrm{HO}_{2}^{-}+\mathrm{O}_{2}$ & 100 & [49] \\
\hline 102 & $\mathrm{O}_{3}+\mathrm{HO}_{2}^{-} \rightarrow \mathrm{O}_{3}^{-}+\mathrm{HO}_{2}$ & $5.50 \times 10^{6}$ & [49] \\
\hline 103 & $\mathrm{O}_{3}+\mathrm{H}_{2} \mathrm{O}_{2} \rightarrow \mathrm{OH}+\mathrm{HO}_{2}+\mathrm{O}_{2}$ & $6.50 \times 10^{-2}$ & [50] \\
\hline 104 & $\mathrm{OH}+\mathrm{O}_{3} \rightarrow \mathrm{HO}_{2}+\mathrm{O}_{2}$ & $1.00 \times 10^{8}$ & [51] \\
\hline 105 & $\mathrm{O}^{-}+\mathrm{O}_{3} \rightarrow \mathrm{O}_{2}^{-}+\mathrm{O}_{2}$ & $1.00 \times 10^{8}$ & [52] \\
\hline 106 & $\mathrm{HO}_{2}+\mathrm{O}_{3} \rightarrow \mathrm{OH}+2 \mathrm{O}_{2}$ & $1.00 \times 10^{4}$ & [51] \\
\hline 107 & $\mathrm{O}_{2}^{-}+\mathrm{O}_{3} \rightarrow \mathrm{O}_{3}^{-}+\mathrm{O}_{2}$ & $1.50 \times 10^{9}$ & [53] \\
\hline 108 & $\mathrm{HO}_{2}+\mathrm{H}_{2} \mathrm{O}_{2} \rightarrow \mathrm{O}_{2}+\mathrm{OH}+\mathrm{H}_{2} \mathrm{O}$ & 0.5 & [54] \\
\hline 109 & $\mathrm{O}_{2}^{-}+\mathrm{H}_{2} \mathrm{O}_{2} \rightarrow \mathrm{OH}^{-}+\mathrm{OH}+\mathrm{O}_{2}$ & 10 & [52] \\
\hline 110 & $\mathrm{O}_{2}^{-}+\mathrm{HO}_{2}^{-} \rightarrow \mathrm{OH}^{-}+\mathrm{O}^{-}+\mathrm{O}_{2}$ & 10 & [52] \\
\hline 111 & $\mathrm{OH}+\mathrm{H}_{2} \mathrm{O}_{2} \rightarrow \mathrm{HO}_{2}+\mathrm{H}_{2} \mathrm{O}$ & $2.65 \times 10^{7}$ & [7] \\
\hline 112 & $\mathrm{OH}+\mathrm{HO}_{2}^{-} \rightarrow \mathrm{O}_{2}^{-}+\mathrm{H}_{2} \mathrm{O}$ & $3.75 \times 10^{9}$ & [7] \\
\hline 113 & $\mathrm{O}^{-}+\mathrm{H}_{2} \mathrm{O}_{2} \rightarrow \mathrm{HO}_{2}+\mathrm{OH}^{-}$ & $3.75 \times 10^{9}$ & [7] \\
\hline 114 & $\mathrm{O}^{-}+\mathrm{HO}_{2}^{-} \rightarrow \mathrm{O}_{2}^{-}+\mathrm{OH}^{-}$ & $6.65 \times 10^{8}$ & [7] \\
\hline 115 & $\mathrm{O}^{-}+\mathrm{O}_{2} \rightarrow \mathrm{O}_{3}^{-}$ & $3.47 \times 10^{9}$ & [7] \\
\hline 116 & $\mathrm{O}_{3}^{-} \rightarrow \mathrm{O}^{-}+\mathrm{O}_{2}$ & $1.90 \times 10^{3}$ & [7] \\
\hline 117 & $\mathrm{OH}+\mathrm{OH} \rightarrow \mathrm{H}_{2} \mathrm{O}_{2}$ & $4.54 \times 10^{9}$ & [7] \\
\hline 118 & $\mathrm{O}^{-}+\mathrm{OH} \rightarrow \mathrm{HO}_{2}^{-}$ & $2.00 \times 10^{9}$ & [52] \\
\hline 119 & $\mathrm{O}^{-}+\mathrm{O}^{-}\left(+\mathrm{H}_{2} \mathrm{O}\right) \rightarrow \mathrm{HO}_{2}^{-}+\mathrm{OH}^{-}$ & $1.00 \times 10^{9}$ & [52] \\
\hline 120 & $\mathrm{OH}+\mathrm{HO}_{2} \rightarrow \mathrm{O}_{2}+\mathrm{H}_{2} \mathrm{O}$ & $8.44 \times 10^{9}$ & [7] \\
\hline 121 & $\mathrm{OH}+\mathrm{O}_{2}^{-} \rightarrow \mathrm{O}_{2}+\mathrm{OH}^{-}$ & $1.02 \times 10^{10}$ & [7] \\
\hline 122 & $\mathrm{O}^{-}+\mathrm{O}_{2}^{-}\left(+\mathrm{H}_{2} \mathrm{O}\right) \rightarrow \mathrm{O}_{2}+2 \mathrm{OH}^{-}$ & $6.00 \times 10^{8}$ & [55] \\
\hline 123 & $\mathrm{OH}+\mathrm{HO}_{3}\left(+\mathrm{H}_{2} \mathrm{O}\right) \rightarrow \mathrm{H}_{2} \mathrm{O}_{2}+\mathrm{O}_{2}$ & $5.00 \times 10^{9}$ & [56] \\
\hline 124 & $\mathrm{OH}+\mathrm{O}_{3}^{-} \rightarrow \mathrm{O}_{2}^{-}+\mathrm{HO}_{2}$ & $6.00 \times 10^{9}$ & [57] \\
\hline 125 & $\mathrm{OH}+\mathrm{O}_{3}^{-} \rightarrow \mathrm{O}_{3}+\mathrm{OH}^{-}$ & $2.50 \times 10^{9}$ & [57] \\
\hline 126 & $\mathrm{O}^{-}+\mathrm{O}_{3}^{-} \rightarrow 2 \mathrm{O}_{2}^{-}$ & $7.00 \times 10^{8}$ & [55] \\
\hline 127 & $\mathrm{HO}_{2}+\mathrm{HO}_{2} \rightarrow \mathrm{H}_{2} \mathrm{O}_{2}+\mathrm{O}_{2}$ & $7.31 \times 10^{5}$ & [7] \\
\hline 128 & $\mathrm{HO}_{2}+\mathrm{O}_{2}^{-}\left(+\mathrm{H}_{2} \mathrm{O}\right) \rightarrow \mathrm{H}_{2} \mathrm{O}_{2}+\mathrm{O}_{2}+\mathrm{OH}^{-}$ & $1.00 \times 10^{8}$ & [7] \\
\hline 129 & $\mathrm{O}_{2}^{-}+\mathrm{O}_{2}^{-}\left(+2 \mathrm{H}_{2} \mathrm{O}\right) \rightarrow \mathrm{H}_{2} \mathrm{O}_{2}+\mathrm{O}_{2}+2 \mathrm{OH}^{-}$ & 0.3 & [7] \\
\hline 130 & $\mathrm{HO}_{2}+\mathrm{HO}_{3} \rightarrow 2 \mathrm{O}_{2}+\mathrm{H}_{2} \mathrm{O}$ & $5.00 \times 10^{9}$ & [56] \\
\hline 131 & $\mathrm{O}_{2}^{-}+\mathrm{HO}_{3} \rightarrow \mathrm{OH}^{-}+2 \mathrm{O}_{2}$ & $1.00 \times 10^{10}$ & [56] \\
\hline 132 & $\mathrm{O}_{2}^{-}+\mathrm{O}_{3}^{-}\left(+\mathrm{H}_{2} \mathrm{O}\right) \rightarrow 2 \mathrm{OH}^{-}+2 \mathrm{O}_{2}$ & $1.00 \times 10^{4}$ & [55] \\
\hline 133 & $\mathrm{HO}_{3}+\mathrm{HO}_{3} \rightarrow \mathrm{H}_{2} \mathrm{O}_{2}+2 \mathrm{O}_{2}$ & $5.00 \times 10^{9}$ & [56] \\
\hline
\end{tabular}




\begin{tabular}{|l|l|l|l|}
\hline 134 & $\mathrm{O}_{3}^{-}+\mathrm{O}_{3}^{-}\left(+\mathrm{H}_{2} \mathrm{O}\right) \rightarrow \mathrm{OH}^{-}+\mathrm{HO}_{2}^{-}+2 \mathrm{O}_{2}$ & $1.00 \times 10^{4}$ & {$[55]$} \\
\hline
\end{tabular}

(A) For the hydrolysis reactions, the values of apparent rate constants $\left(\mathrm{s}^{-1}\right)$ are given. They are equal to the product of the true bimolecular rate constant on water concentration $55.5 \mathrm{~mol} / \mathrm{L}$.

(B) $k_{33}\left(\mathrm{~L} \mathrm{~mol}^{-1} \mathrm{~s}^{-1}\right)=\frac{2.20 \times 10^{-3}+0.0193\left[\mathrm{H}^{+}\right]}{1+0.0650\left[\mathrm{H}^{+}\right]\left[\mathrm{Cl}^{-}\right]}$

(C) The values of constants $k_{34}, k_{35}, k_{61}$ have been varied to bring the calculation results closer to the experimental data.

(D) $k_{76}\left(\mathrm{~L} \mathrm{~mol}^{-1} \mathrm{~s}^{-1}\right)=\frac{183.3}{1+2.27\left[\mathrm{H}^{+}\right]\left[\mathrm{Cl}^{-}\right]}$

Table S3. Equilibrium and Rate Constants of Phosphates Acid Dissociation at 20 ${ }^{\circ} \mathrm{C}$ and ionic strength $1 \mathrm{M}$.

\begin{tabular}{|l|l|l|}
\hline Reaction & Equilibrium constant & Ref \\
\hline $\mathrm{H}_{3} \mathrm{PO}_{4} \rightleftarrows \mathrm{H}^{+}+\mathrm{H}_{2} \mathrm{PO}_{4}^{--}$ & $2.4 \times 10^{-2}$ & {$[58]$} \\
\hline $\mathrm{H}_{2} \mathrm{PO}_{4}{ }^{-} \rightleftarrows \mathrm{H}^{+}+\mathrm{HPO}_{4}{ }^{2-}$ & $8.0 \times 10^{-7}$ & {$[58]$} \\
\hline $\mathrm{HPO}_{4}{ }^{2-} \rightleftarrows \mathrm{H}^{+}+\mathrm{PO}_{4}{ }^{3-}$ & $1.5 \times 10^{-9}$ & {$[58]$} \\
\hline Reaction & Rate constant & \\
\hline $\mathrm{H}_{3} \mathrm{PO}_{4} \rightarrow \mathrm{H}^{+}+\mathrm{H}_{2} \mathrm{PO}_{4}^{-}$ & $1.2 \times 10^{9}$ & \\
\hline $\mathrm{H}^{+}+\mathrm{H}_{2} \mathrm{PO}_{4}^{-} \rightarrow \mathrm{H}_{3} \mathrm{PO}_{4}$ & $5 \times 10^{10}$ & \\
\hline $\mathrm{H}_{2} \mathrm{PO}_{4}^{-} \rightarrow \mathrm{H}^{+}+\mathrm{HPO}_{4}{ }^{2-}$ & $4.0 \times 10^{4}$ & \\
\hline $\mathrm{H}^{+}+\mathrm{HPO}_{4}{ }^{2-} \rightarrow \mathrm{H}_{2} \mathrm{PO}_{4}^{-}$ & $5 \times 10^{10}$ & \\
\hline $\mathrm{HPO}_{4}{ }^{2-} \rightarrow \mathrm{H}^{+}+\mathrm{PO}_{4}{ }^{3-}$ & 75 & \\
\hline $\mathrm{H}^{+}+\mathrm{PO}_{4}{ }^{3-} \rightarrow \mathrm{HPO}_{4}{ }^{2-}$ & $5 \times 10^{10}$ & \\
\hline
\end{tabular}



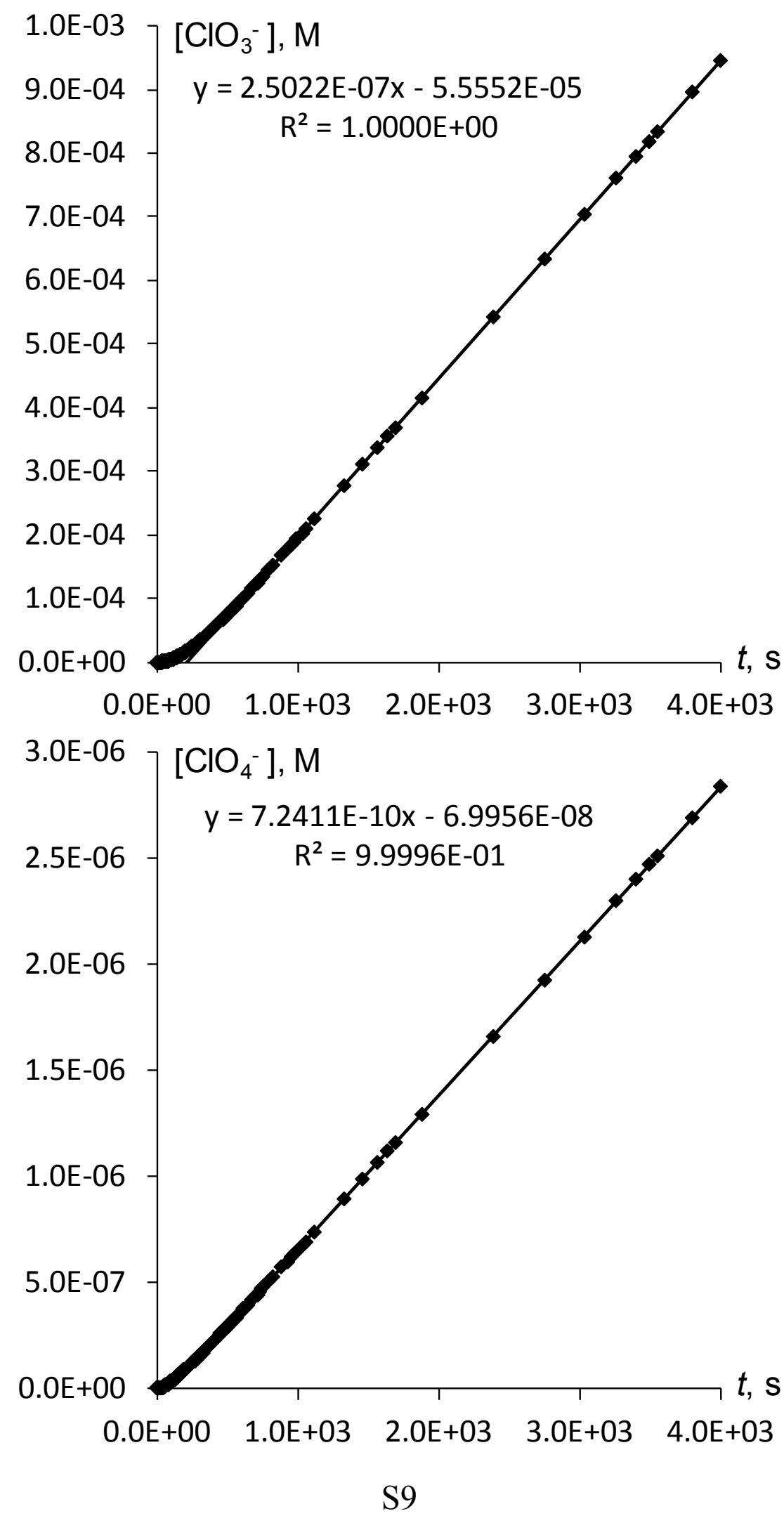


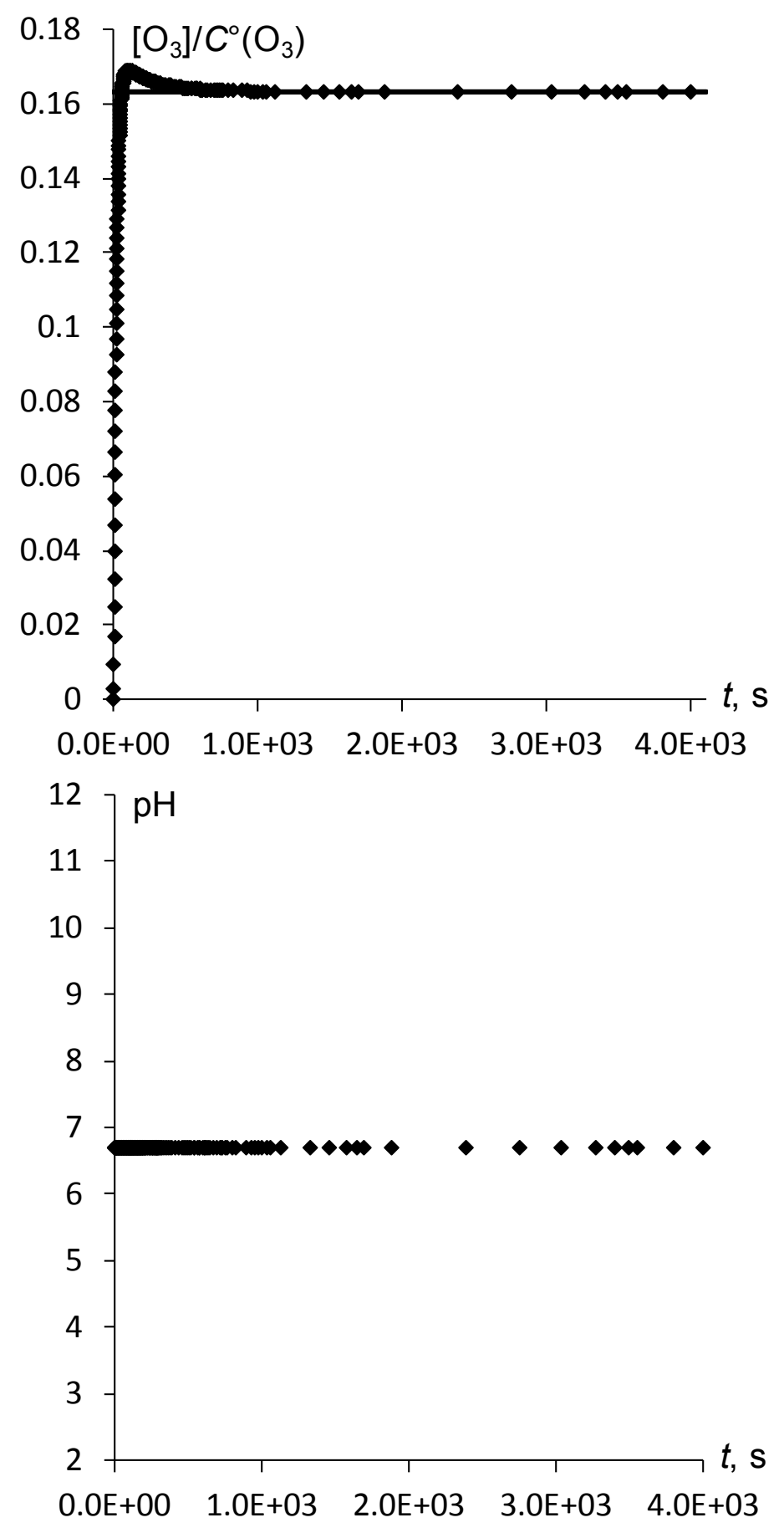

Fig. S1. Time dependencies of chlorate and perchlorate concentrations, ozone solubility coefficient, and $\mathrm{pH}$ of reaction solution calculated by the model with the initial set of chemical reactions and corrected values of rate constants $k_{34}, k_{35}, k_{61}$. Initial $\mathrm{pH}$ 6.7, other parameters of the calculations are indicated in the text of the article and are the same as when calculating chlorate formation rate. 


\section{REDUCED SET OF CHEMICAL REACTIONS IN THE SYSTEM O $\mathrm{O}_{3}-\mathrm{Cl}^{-}$ IN AQUEOUS SOLUTION}

Table S4. Reactions Included in the Reduced Set and their Rate Constants

Note: Also reactions of phosphates acid dissociation (Table S3) were used in the calculations with $\mathrm{pH}<7$.

\begin{tabular}{|c|c|c|c|}
\hline No. & Reaction & Rate Constants & Ref \\
\hline 1 & $\mathrm{O}_{2}($ gas $) \rightarrow 0.055 \mathrm{O}_{2}(\mathrm{aq})$ & 0.0063 & {$[1]$} \\
\hline 2 & $\mathrm{O}_{2}(\mathrm{aq}) \rightarrow 18.2 \mathrm{O}_{2}(\mathrm{gas})$ & 0.1 & [17] \\
\hline 3 & $\mathrm{O}_{3}(\mathrm{gas}) \rightarrow 0.055 \mathrm{O}_{3}(\mathrm{aq})$ & 0.33 & [2] \\
\hline 4 & $\mathrm{O}_{3}(\mathrm{aq}) \rightarrow 18.2 \mathrm{O}_{3}(\mathrm{gas})$ & 0.1 & [17] \\
\hline 5 & $\mathrm{Cl}_{2}($ gas $) \rightarrow 0.055 \mathrm{Cl}_{2}(\mathrm{aq})$ & 2.8 & [3] \\
\hline 6 & $\mathrm{Cl}_{2}(\mathrm{aq}) \rightarrow 18.2 \mathrm{Cl}_{2}(\mathrm{gas})$ & 0.1 & [17] \\
\hline 7 & $\mathrm{H}_{2} \mathrm{O} \rightarrow \mathrm{H}^{+}+\mathrm{OH}^{-}$ & $6.72 \times 10^{-4}$ & [7] \\
\hline 8 & $\mathrm{H}^{+}+\mathrm{OH}^{-} \rightarrow \mathrm{H}_{2} \mathrm{O}$ & $1.00 \times 10^{11}$ & [7] \\
\hline 9 & $\mathrm{H}_{2} \mathrm{O}_{2} \rightarrow \mathrm{H}^{+}+\mathrm{HO}_{2}^{-}$ & $7.18 \times 10^{-2}$ & [7] \\
\hline 10 & $\mathrm{H}^{+}+\mathrm{HO}_{2}^{-} \rightarrow \mathrm{H}_{2} \mathrm{O}_{2}$ & $5 \times 10^{10}$ & \\
\hline 11 & $\mathrm{OH}+\mathrm{OH}^{-} \rightarrow \mathrm{O}^{-}+\mathrm{H}_{2} \mathrm{O}$ & $1.17 \times 10^{10}$ & [7] \\
\hline 12 & $\mathrm{O}^{-}+\mathrm{H}_{2} \mathrm{O} \rightarrow \mathrm{OH}+\mathrm{OH}^{-}$ & $3.84 \times 10^{7}$ & [8] \\
\hline 13 & $\mathrm{HO}_{2} \rightarrow \mathrm{H}^{+}+\mathrm{O}_{2}^{-}$ & $6.62 \times 10^{5}$ & [7] \\
\hline 14 & $\mathrm{H}^{+}+\mathrm{O}_{2}^{-} \rightarrow \mathrm{HO}_{2}$ & $4.52 \times 10^{10}$ & [7] \\
\hline 15 & $\mathrm{HOCl} \rightarrow \mathrm{H}^{+}+\mathrm{ClO}^{-}$ & $1.99 \times 10^{3}$ & {$[11,59]$} \\
\hline 16 & $\mathrm{H}^{+}+\mathrm{ClO}^{-} \rightarrow \mathrm{HOCl}$ & $5 \times 10^{10}$ & \\
\hline 17 & $\mathrm{Cl}_{2}\left(+\mathrm{H}_{2} \mathrm{O}\right) \rightarrow \mathrm{H}^{+}+\mathrm{Cl}^{-}+\mathrm{HOCl}$ & $22(\mathrm{~A})$ & [14] \\
\hline 18 & $\mathrm{H}^{+}+\mathrm{Cl}^{-}+\mathrm{HOCl} \rightarrow \mathrm{Cl}_{2}+\mathrm{H}_{2} \mathrm{O}$ & $2.14 \times 10^{4}$ & [14] \\
\hline 19 & $\mathrm{O}_{3}+\mathrm{Cl}^{-} \rightarrow \mathrm{ClO}^{-}+\mathrm{O}_{2}$ & $2.2 \times 10^{-3}$ & [2] \\
\hline 20 & $\mathrm{ClO}^{-}+\mathrm{O}_{3} \rightarrow \mathrm{Cl}^{-}+2 \mathrm{O}_{2}$ & $60\left({ }^{\mathrm{B}}\right)$ & [19] \\
\hline 21 & $\mathrm{ClO}^{-}+\mathrm{O}_{3} \rightarrow \mathrm{ClO}_{2}^{-}+\mathrm{O}_{2}$ & $30\left({ }^{\mathrm{B}}\right)$ & [19] \\
\hline 22 & $\mathrm{ClO}_{2}^{-}+\mathrm{O}_{3} \rightarrow \mathrm{ClO}_{2}+\mathrm{O}_{3}^{-}$ & $8.20 \times 10^{6}$ & {$[20,21]$} \\
\hline 23 & $\mathrm{ClO}_{2}+\mathrm{O}_{3} \rightarrow \mathrm{ClO}_{3}+\mathrm{O}_{2}$ & $1.40 \times 10^{3}$ & [24] \\
\hline 24 & $\mathrm{Cl}^{-}+\mathrm{OH} \rightarrow \mathrm{ClOH}^{-}$ & $4.20 \times 10^{9}$ & [27] \\
\hline 25 & $\mathrm{ClOH}^{-} \rightarrow \mathrm{Cl}^{-}+\mathrm{OH}$ & $6.10 \times 10^{9}$ & {$[27]$} \\
\hline 26 & $\mathrm{ClOH}^{-} \rightarrow \mathrm{Cl}+\mathrm{OH}^{-}$ & 23 & {$[28,29]$} \\
\hline
\end{tabular}




\begin{tabular}{|c|c|c|c|}
\hline 27 & $\mathrm{Cl}+\mathrm{OH}^{-} \rightarrow \mathrm{ClOH}^{-}$ & $1.80 \times 10^{10}$ & {$[28,29]$} \\
\hline 28 & $\mathrm{ClOH}^{-}+\mathrm{H}^{+} \rightarrow \mathrm{Cl}+\mathrm{H}_{2} \mathrm{O}$ & $2.40 \times 10^{10}$ & [27] \\
\hline 29 & $\mathrm{Cl}\left(+\mathrm{H}_{2} \mathrm{O}\right) \rightarrow \mathrm{ClOH}^{-}+\mathrm{H}^{+}$ & $1.80 \times 10^{5}(\mathrm{~A})$ & [27] \\
\hline 30 & $\mathrm{Cl}+\mathrm{Cl}^{-} \rightarrow \mathrm{Cl}_{2}^{-}$ & $7.80 \times 10^{9}$ & [27] \\
\hline 31 & $\mathrm{Cl}_{2}^{-} \rightarrow \mathrm{Cl}+\mathrm{Cl}^{-}$ & $5.70 \times 10^{4}$ & [27] \\
\hline 32 & $\mathrm{Cl}_{2}^{-}\left(+\mathrm{H}_{2} \mathrm{O}\right) \rightarrow \mathrm{ClOH}^{-}+\mathrm{H}^{+}+\mathrm{Cl}^{-}$ & $1.30 \times 10^{3}\left({ }^{\mathrm{A}}\right)$ & [27] \\
\hline 33 & $\mathrm{Cl}^{-}+\mathrm{ClOH}^{-} \rightarrow \mathrm{Cl}_{2}^{-}+\mathrm{OH}^{-}$ & $1.00 \times 10^{4}$ & [30] \\
\hline 34 & $\mathrm{Cl}_{2}^{-}+\mathrm{OH}^{-} \rightarrow \mathrm{Cl}^{-}+\mathrm{ClOH}^{-}$ & $4.50 \times 10^{7}$ & [30] \\
\hline 35 & $\mathrm{Cl}_{2}^{-}+\mathrm{Cl}_{2}^{-} \rightarrow \mathrm{Cl}_{2}+2 \mathrm{Cl}^{-}$ & $3.50 \times 10^{9}$ & [27] \\
\hline 36 & $\mathrm{Cl}_{2}^{-}+\mathrm{O}_{3} \rightarrow \mathrm{ClO}+\mathrm{Cl}^{-}+\mathrm{O}_{2}$ & $1.5 \times 10^{7}(\mathrm{~B})$ & [33] \\
\hline 37 & $\mathrm{HOCl}+\mathrm{OH} \rightarrow \mathrm{ClO}+\mathrm{H}_{2} \mathrm{O}$ & $1.20 \times 10^{8}$ & [22] \\
\hline 38 & $\mathrm{ClO}^{-}+\mathrm{OH} \rightarrow \mathrm{ClO}+\mathrm{OH}^{-}$ & $3.00 \times 10^{9}$ & [22] \\
\hline 39 & $\mathrm{ClO}^{-}+\mathrm{O}^{-}\left(+\mathrm{H}_{2} \mathrm{O}\right) \rightarrow \mathrm{ClO}+2 \mathrm{OH}^{-}$ & $2.30 \times 10^{8}$ & [25] \\
\hline 40 & $\mathrm{ClO}+\mathrm{O}_{3}^{-} \rightarrow \mathrm{ClO}^{-}+\mathrm{O}_{3}$ & $1.00 \times 10^{9}$ & [36] \\
\hline 41 & $\mathrm{ClO}+\mathrm{ClO} \rightarrow \mathrm{Cl}_{2} \mathrm{O}_{2}$ & $2.5 \times 10^{9}$ & [28] \\
\hline 42 & $\mathrm{ClO}+\mathrm{ClO}_{2} \rightarrow \mathrm{ClOClO}_{2}$ & $7.4 \times 10^{9}$ & [48] \\
\hline 43 & $\mathrm{ClO}+\mathrm{ClO}_{3} \rightarrow \mathrm{ClOClO}_{3}$ & $7.4 \times 10^{9}$ & [48] \\
\hline 44 & $\mathrm{ClO}_{2}+\mathrm{ClO}_{3} \rightarrow \mathrm{O}_{2} \mathrm{ClOClO}_{2}$ & $7.4 \times 10^{9}$ & [48] \\
\hline 45 & $\mathrm{Cl}_{2} \mathrm{O}_{2}\left(+\mathrm{H}_{2} \mathrm{O}\right) \rightarrow \mathrm{Cl}^{-}+\mathrm{ClO}_{3}^{-}+2 \mathrm{H}^{+}$ & $1 \times 10^{4}\left({ }^{\mathrm{A}}\right)$ & [48] \\
\hline 46 & $\mathrm{ClOClO}_{2}\left(+\mathrm{H}_{2} \mathrm{O}\right) \rightarrow \mathrm{HOCl}+\mathrm{ClO}_{3}^{-}+\mathrm{H}^{+}$ & $1 \times 10^{4}\left({ }^{\mathrm{A}}\right)$ & [48] \\
\hline 47 & $\mathrm{ClOClO}_{3}\left(+\mathrm{H}_{2} \mathrm{O}\right) \rightarrow \mathrm{HOCl}+\mathrm{ClO}_{4}^{-}+\mathrm{H}^{+}$ & $1 \times 10^{4}\left({ }^{\mathrm{A}}\right)$ & [48] \\
\hline 48 & $\mathrm{O}_{2} \mathrm{ClOClO}_{2}\left(+\mathrm{H}_{2} \mathrm{O}\right) \rightarrow 2 \mathrm{ClO}_{3}^{-}+2 \mathrm{H}^{+}$ & $1 \times 10^{4}\left({ }^{\mathrm{A}}\right)$ & [48] \\
\hline 49 & $\mathrm{O}_{3}+\mathrm{OH}^{-} \rightarrow \mathrm{HO}_{2}^{-}+\mathrm{O}_{2}$ & 100 & [49] \\
\hline 50 & $\mathrm{O}_{3}+\mathrm{HO}_{2}^{-} \rightarrow \mathrm{O}_{3}^{-}+\mathrm{HO}_{2}$ & $5.50 \times 10^{6}$ & [49] \\
\hline 51 & $\mathrm{OH}+\mathrm{O}_{3} \rightarrow \mathrm{HO}_{2}+\mathrm{O}_{2}$ & $1.00 \times 10^{8}$ & [51] \\
\hline 52 & $\mathrm{O}^{-}+\mathrm{O}_{3} \rightarrow \mathrm{O}_{2}^{-}+\mathrm{O}_{2}$ & $1.00 \times 10^{8}$ & [52] \\
\hline 53 & $\mathrm{O}_{2}^{-}+\mathrm{O}_{3} \rightarrow \mathrm{O}_{3}^{-}+\mathrm{O}_{2}$ & $1.50 \times 10^{9}$ & [53] \\
\hline 54 & $\mathrm{H}^{+}+\mathrm{O}_{3}^{-} \rightarrow \mathrm{HO}_{3}$ & $5.20 \times 10^{10}$ & [9] \\
\hline 55 & $\mathrm{HO}_{3} \rightarrow \mathrm{OH}+\mathrm{O}_{2}$ & $1.10 \times 10^{5}$ & [9] \\
\hline 56 & $\mathrm{OH}+\mathrm{H}_{2} \mathrm{O}_{2} \rightarrow \mathrm{HO}_{2}+\mathrm{H}_{2} \mathrm{O}$ & $2.65 \times 10^{7}$ & [7] \\
\hline 57 & $\mathrm{OH}+\mathrm{HO}_{2}^{-} \rightarrow \mathrm{O}_{2}^{-}+\mathrm{H}_{2} \mathrm{O}$ & $3.75 \times 10^{9}$ & [7] \\
\hline 58 & $\mathrm{O}^{-}+\mathrm{H}_{2} \mathrm{O}_{2} \rightarrow \mathrm{HO}_{2}+\mathrm{OH}^{-}$ & $3.75 \times 10^{9}$ & [7] \\
\hline 59 & $\mathrm{O}^{-}+\mathrm{HO}_{2}^{-} \rightarrow \mathrm{O}_{2}^{-}+\mathrm{OH}^{-}$ & $6.65 \times 10^{8}$ & [7] \\
\hline
\end{tabular}




\begin{tabular}{|l|l|l|l|}
\hline 60 & $\mathrm{O}^{-}+\mathrm{O}_{2} \rightarrow \mathrm{O}_{3}^{-}$ & $3.47 \times 10^{9}$ & {$[7]$} \\
\hline 61 & $\mathrm{O}_{3}^{-} \rightarrow \mathrm{O}^{-}+\mathrm{O}_{2}$ & $1.90 \times 10^{3}$ & {$[7]$} \\
\hline 62 & $\mathrm{OH}+\mathrm{OH} \rightarrow \mathrm{H}_{2} \mathrm{O}_{2}$ & $4.54 \times 10^{9}$ & {$[7]$} \\
\hline 63 & $\mathrm{O}^{-}+\mathrm{OH} \rightarrow \mathrm{HO}_{2}^{-}$ & $2.00 \times 10^{9}$ & {$[52]$} \\
\hline 64 & $\mathrm{O}^{-}+\mathrm{O}^{-}\left(+\mathrm{H}_{2} \mathrm{O}\right) \rightarrow \mathrm{HO}_{2}{ }^{-}+\mathrm{OH}^{-}$ & $1.00 \times 10^{9}$ & {$[52]$} \\
\hline 65 & $\mathrm{OH}+\mathrm{HO}_{2} \rightarrow \mathrm{O}_{2}+\mathrm{H}_{2} \mathrm{O}$ & $8.44 \times 10^{9}$ & {$[7]$} \\
\hline 66 & $\mathrm{OH}+\mathrm{O}_{2}^{-} \rightarrow \mathrm{O}_{2}+\mathrm{OH}^{-}$ & $1.02 \times 10^{10}$ & {$[7]$} \\
\hline 67 & $\mathrm{O}^{-}+\mathrm{O}_{2}^{-}\left(+\mathrm{H}_{2} \mathrm{O}\right) \rightarrow \mathrm{O}_{2}+2 \mathrm{OH}^{-}$ & $6.00 \times 10^{8}$ & {$[55]$} \\
\hline
\end{tabular}

(A) For the hydrolysis reactions, the values of apparent rate constants $\left(\mathrm{s}^{-1}\right)$ are given. They are equal to the product of the true bimolecular rate constant on water concentration $55.5 \mathrm{~mol} / \mathrm{L}$.

(B) The constants $k_{20}, k_{21}, k_{36}$ have been changed from their original values in order to bring the calculation results closer to the experimental data. 


\section{THE EFFECT OF UNCERTAINTY IN THE RATE CONSTANT OF REACTION $\mathrm{O}_{3}+\mathrm{OH}^{-} \rightarrow \mathrm{HO}_{2}^{-}+\mathrm{O}_{2}$ ON THE FITTED VALUE OF THE RATE CONSTANT OF $\mathrm{Cl}_{2}{ }^{-}+\mathrm{O}_{3} \rightarrow \mathrm{ClO}^{\circ}+\mathrm{Cl}^{-}+\mathrm{O}_{2}$ REACTION}

In our model we evaluated the corrected value for the rate constant of reaction

$$
\mathrm{Cl}_{2}{ }^{-}+\mathrm{O}_{3} \rightarrow \mathrm{ClO}^{\cdot}+\mathrm{Cl}^{-}+\mathrm{O}_{2}
$$

from the criterion that the calculated and experimental chlorate formation rates coincide at the maximum at $\mathrm{pH} 10$. In this section, the reactions are numbered as in the main text of the article.

It is understandable that the fitted value of $k_{4}$ is affected by the adopted values of rate constants of other reactions in the mechanism. In particular, the influence of the rate constant of reaction

$$
\mathrm{O}_{3}+\mathrm{OH}^{-} \rightarrow \mathrm{HO}_{2}^{-}+\mathrm{O}_{2}
$$

is significant. This is because reaction (5) is the initiation stage of ozone selfdecomposition complex reaction, which is the source of hydroxyl free radicals for chloride ion oxidation. Since both reactions (4) and (5) are parts of the reaction chain that leads to chlorate generation, the fitted value of $k_{4}$ is in inverse relation with the adopted value of $k_{5}$. The relationship between them is demonstrated if Fig. S2 (on the next page), and is described by the equation

$$
\frac{9.52 \times 10^{6}}{k_{4}}+\frac{37.8}{k_{5}}=1
$$

From Eq. (S1) follow the necessary conditions on the magnitudes of $k_{4}$ and $k_{5}$ :

$$
k_{4} \gtrsim 9.5 \times 10^{6} \mathrm{~L} \mathrm{~mol}^{-1} \mathrm{~s}^{-1}, \quad k_{5} \gtrsim 38 \mathrm{~L} \mathrm{~mol}^{-1} \mathrm{~s}^{-1} .
$$

They agree with the ranges of these constants values from the literature:

$$
k_{4} \leq 9 \times 10^{7} \mathrm{~L} \mathrm{~mol}^{-1} \mathrm{~s}^{-1}[33], \quad 40 \leq k_{5} \leq 220 \mathrm{~L} \mathrm{~mol}^{-1} \mathrm{~s}^{-1}[49,60-64] .
$$

The uncertainty in the available values of $k_{4}$ and $k_{5}$ stresses the need for further studies of these important reactions. 


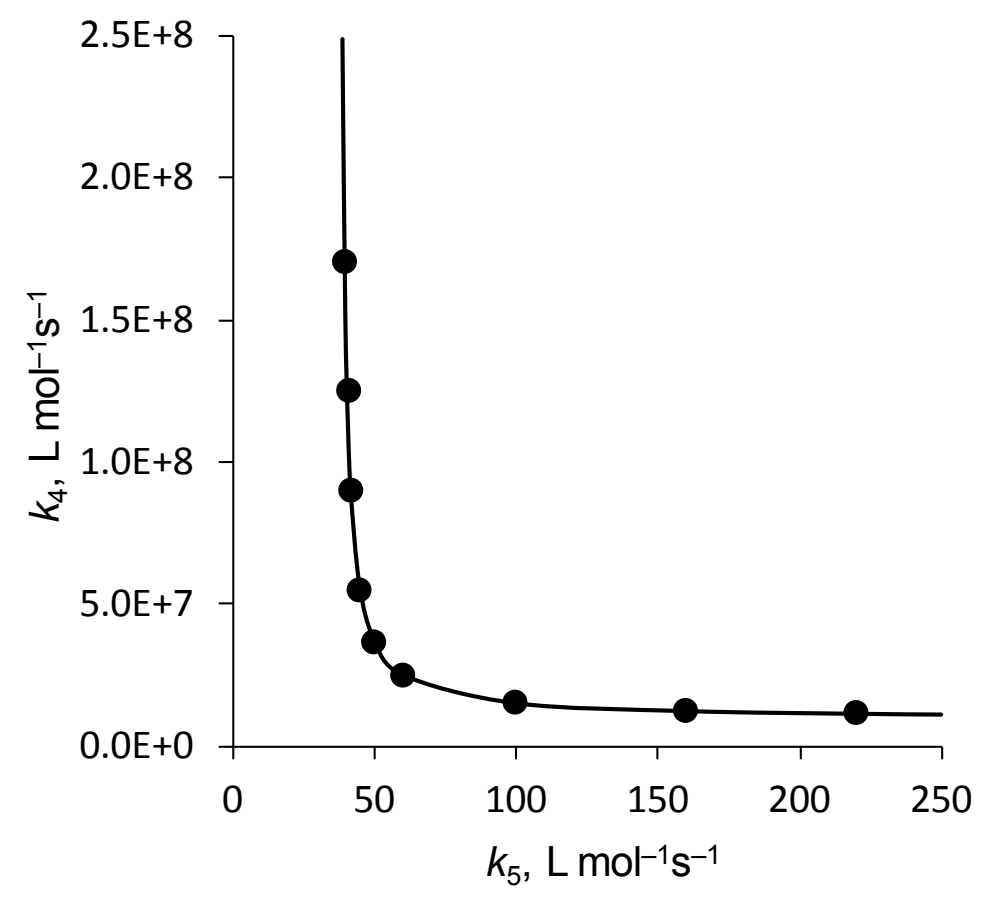

Fig. S2. The relationship between the adopted values of $k_{5}$ and the fitted values of $k_{4}$ obtained from the criterion of equality of experimental and calculated chlorate formation rates at $\mathrm{pH} 10, C^{\circ}\left(\mathrm{O}_{3}\right)=95 \mathrm{~g} / \mathrm{m}^{3}$, temperature $20^{\circ} \mathrm{C}$, ionic strength and chloride ion concentration $1 \mathrm{M}, k_{L} a=0.1 \mathrm{~s}^{-1}, V_{\text {liq }}=0.25 \mathrm{~L}, V_{\text {gas }}=0.055 \cdot V_{\text {liq }}, v=21$ $\mathrm{L} / \mathrm{h}=5.83 \cdot 10^{-3} \mathrm{~L} / \mathrm{s}$.

Points represent the values obtained by fitting the chlorate formation rates. Line depicts the relationship between $k_{4}$ and $k_{5}$ described by Eq. (S1). 


\section{KINETIC ORDER OF CHLORATE FORMATION REACTION WITH RESPECT TO OZONE AND CHLORIDE}

When determining the kinetic order of chlorate formation reaction, it is assumed that chlorate formation rate, $d\left[\mathrm{ClO}_{3}{ }^{-}\right] / d t$, is obeyed to the kinetic law

$$
d\left[\mathrm{ClO}_{3}^{-}\right] / d t=k\left(\mathrm{C}^{\circ}\left(\mathrm{O}_{3}\right)\right)^{\alpha}\left[\mathrm{Cl}^{-}\right]^{\beta},
$$

where $C^{\circ}\left(\mathrm{O}_{3}\right)$ is ozone concentration in gas flow at entrance to the reactor, $\left[\mathrm{Cl}^{-}\right]$is chloride ion concentration in reaction solution, $\alpha$ and $\beta$ are the kinetic orders with respect to correspondingly $\mathrm{O}_{3}$ (gas) and $\mathrm{Cl}^{-}$, and $k$ is apparent rate constant.

If the concentration of ozone is varied and that one of chloride is constant, then the kinetic law in double logarithmic coordinates should represent a straight line:

$$
\log _{10}\left(d\left[\mathrm{ClO}_{3}^{-}\right] / d t\right)=\text { const }_{1}+\alpha \cdot \log _{10} C^{\circ}\left(\mathrm{O}_{3}\right),
$$

where const ${ }_{1} \equiv \log _{10}\left(k\left[\mathrm{Cl}^{-}\right]^{\beta}\right)$. The slope of the line gives the value of the order $\alpha$. Similarly, if chloride concentration is varied and that of ozone is kept constant, then the kinetic law takes the form

$$
\log _{10}\left(d\left[\mathrm{ClO}_{3}^{-}\right] / d t\right)=\text { const }_{2}+\beta \cdot \log _{10}\left[\mathrm{Cl}^{-}\right],
$$

where const $t_{2} \equiv \log _{10}\left(k\left(C^{\circ}\left(\mathrm{O}_{3}\right)\right)^{\alpha}\right)$, and the line slope equals to $\beta$.

It can be seen from Fig. S3 that the experimental and calculated dependencies of $\log _{10}\left(d\left[\mathrm{ClO}_{3}{ }^{-}\right] / d t\right)$ versus $\log _{10} \mathrm{C}^{\circ}\left(\mathrm{O}_{3}\right)\left(\right.$ at $\left[\mathrm{Cl}^{-}\right]=1 \mathrm{M}$ and $\left.\mathrm{pH} 6\right)$ and $\log _{10}\left(d\left[\mathrm{ClO}_{3}^{-}\right] / d t\right)$ versus $\log _{10}\left[\mathrm{Cl}^{-}\right]\left(\right.$at $C^{\circ}\left(\mathrm{O}_{3}\right)=95 \mathrm{~g} / \mathrm{m}^{3}$ and $\left.\mathrm{pH} 6\right)$ agree quite well and meet correspondingly the values of kinetic orders $\alpha \approx 1$ and $\beta \approx 1$. Thus the mechanism and the model adequately render the empirical kinetic laws of chlorate formation found in ref [65]. 

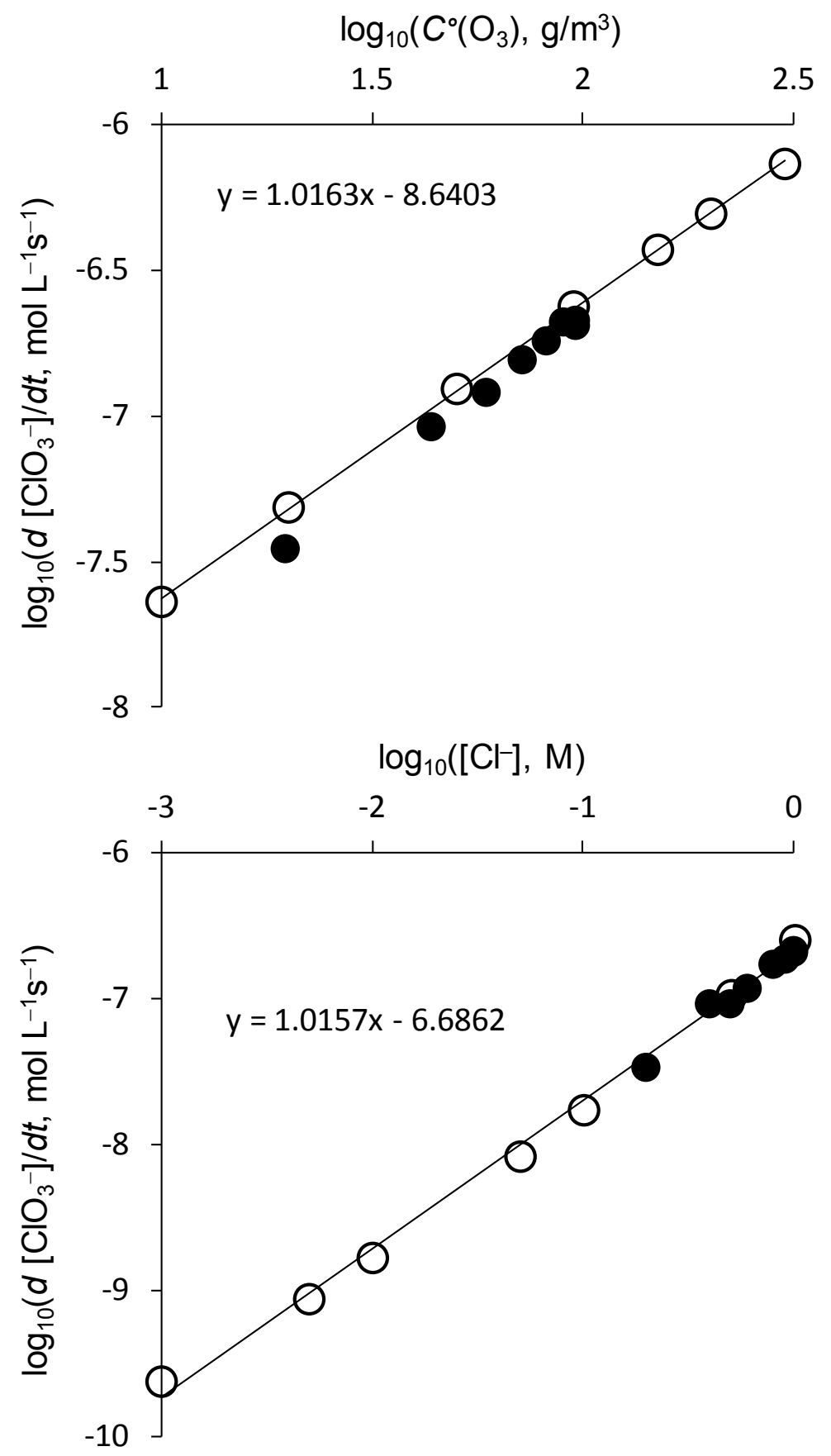

Fig. S3. Dependencies of chlorate formation rate on ozone concentration in initial gases at $\left[\mathrm{Cl}^{-}\right]=1 \mathrm{M}$ (upper panel) and on chloride concentration in reaction solution at $C^{\circ}\left(\mathrm{O}_{3}\right)=95 \mathrm{~g} / \mathrm{m}^{3}$ (lower panel).

Solid dots are the experimental data [65] obtained at temperature $20 \pm 1{ }^{\circ} \mathrm{C}$, ionic strength $1 \mathrm{M}, \mathrm{pH} \sim 6$.

Empty dots connected with straight lines are results of calculations by the model with reduced reaction set at temperature $20^{\circ} \mathrm{C}$, ionic strength $1 \mathrm{M}, \mathrm{pH}=6$. 


\section{EXPERIMENTAL AND CALCULATED RATES OF PERCHLORATE FORMATION ON OZONATION OF CHLORIDE SOLUTIONS}

Table S5. Experimental and Calculated Rates of Perchlorate Formation, $\mathrm{mol} \mathrm{L}^{-1} \mathrm{~s}^{-1}$, on Ozonation of $\mathrm{NaCl}$ Solutions

\begin{tabular}{|l|l|l|l|l|l|l|l|}
\hline No. & $\begin{array}{l}{\left[\mathrm{Cl}^{-}\right],} \\
\mathrm{mM}\end{array}$ & $\begin{array}{l}\mathrm{C}^{\circ}\left(\mathrm{O}_{3}\right), \\
\mathrm{g} / \mathrm{m}^{3}\end{array}$ & $\mathrm{pH}$ & $\begin{array}{l}r_{\mathrm{ClO} 4-}, \\
\text { experiment } \\
{[66,67]}\end{array}$ & $\begin{array}{l}r_{\mathrm{ClO} 4}, \\
\text { calculated } \\
(1)\end{array}$ & $\begin{array}{l}r_{\mathrm{ClO} 4}, \\
\text { calculated } \\
(2)\end{array}$ & $\begin{array}{l}r_{\mathrm{ClO} 4}, \\
\text { calculated } \\
(3)\end{array}$ \\
\hline 1 & 0.28 & 24 & 5.6 & $4.83 \times 10^{-14}$ & $3.06 \times 10^{-13}$ & $2.77 \times 10^{-13}$ & $1.17 \times 10^{-14}$ \\
\hline 2 & 2.8 & 3.36 & 5.6 & $1.33 \times 10^{-14}$ & $8.86 \times 10^{-14}$ & $8.40 \times 10^{-14}$ & $6.43 \times 10^{-15}$ \\
\hline 3 & 2.8 & 149 & 5.9 & $3.5 \times 10^{-13}$ & $2.87 \times 10^{-11}$ & $2.68 \times 10^{-11}$ & $1.85 \times 10^{-12}$ \\
\hline 4 & 2.8 & 33.6 & 6.5 & $8.83 \times 10^{-14}$ & $5.00 \times 10^{-13}$ & $4.54 \times 10^{-13}$ & $7.24 \times 10^{-14}$ \\
\hline 5 & 3.35 & 18.8 & 6.5 & $3.8 \times 10^{-13}$ & $1.07 \times 10^{-12}$ & $9.75 \times 10^{-13}$ & $1.48 \times 10^{-13}$ \\
\hline 6 & 3.36 & 18.8 & 6.5 & $3.8 \times 10^{-13}$ & $1.07 \times 10^{-12}$ & $9.75 \times 10^{-13}$ & $1.48 \times 10^{-13}$ \\
\hline 7 & 28 & 33.6 & 6.5 & $4.0 \times 10^{-13}$ & $1.17 \times 10^{-11}$ & $1.03 \times 10^{-11}$ & $3.02 \times 10^{-12}$ \\
\hline
\end{tabular}

Experimental conditions [66, 67] were as follows: duration of ozonation 250 hours [67] (No. 1, 2, 3, 4, 7), 170 hours [66] (No. 5, 6), flow rate of ozonized oxygen 0.3 $\mathrm{L} / \mathrm{h}$ (STP), volume of the reaction solution $500 \mathrm{~mL}$ [67] (No. 1, 2, 3, 4, 7), $100 \mathrm{~mL}$ [66] (No. 5, 6).

The calculated rates are obtained with the following sets of chemical reactions:

(1) - the initial set with corrected rate constants of reactions

$\mathrm{ClO}^{-}+\mathrm{O}_{3} \rightarrow \mathrm{Cl}^{-}+2 \mathrm{O}_{2}, \mathrm{ClO}^{-}+\mathrm{O}_{3} \rightarrow \mathrm{ClO}_{2}^{-}+\mathrm{O}_{2}, \mathrm{Cl}_{2}^{-}+\mathrm{O}_{3} \rightarrow \mathrm{ClO}+\mathrm{Cl}^{-}+\mathrm{O}_{2}$;

(2) - the reduced set with corrected rate constants of reactions

$\mathrm{ClO}^{-}+\mathrm{O}_{3} \rightarrow \mathrm{Cl}^{-}+2 \mathrm{O}_{2}, \mathrm{ClO}^{-}+\mathrm{O}_{3} \rightarrow \mathrm{ClO}_{2}^{-}+\mathrm{O}_{2}, \mathrm{Cl}_{2}^{-}+\mathrm{O}_{3} \rightarrow \mathrm{ClO}+\mathrm{Cl}^{-}+\mathrm{O}_{2}$;

(3) - the reduced set with corrected rate constants of reactions

$\mathrm{ClO}^{-}+\mathrm{O}_{3} \rightarrow \mathrm{Cl}^{-}+2 \mathrm{O}_{2}, \mathrm{ClO}^{-}+\mathrm{O}_{3} \rightarrow \mathrm{ClO}_{2}^{-}+\mathrm{O}_{2}, \mathrm{Cl}_{2}^{-}+\mathrm{O}_{3} \rightarrow \mathrm{ClO}+\mathrm{Cl}^{-}+\mathrm{O}_{2}$, and with the addition of reaction $\mathrm{ClO}_{3}{ }^{\circ}+\mathrm{O}_{3} \rightarrow \mathrm{ClO}_{2}{ }^{\circ}+2 \mathrm{O}_{2}$ with rate constant $2 \times 10^{5} \mathrm{~L} \mathrm{~mol}^{-1} \mathrm{~s}^{-1}$. 


\section{ESTIMATION OF STANDARD GIBBS ENERGY OF REACTION $\mathrm{ClO}_{3}{ }^{\circ}+\mathrm{O}_{3} \rightarrow \mathrm{ClO}_{2}{ }^{\circ}+2 \mathrm{O}_{2}$ IN AQUEOUS SOLUTION}

The standard electrode potential of the half reaction $\mathrm{ClO}_{3}{ }^{-}+\overline{\mathrm{e}} \rightarrow \mathrm{ClO}_{3}^{-}$was determined in ref [68]:

$$
E^{\circ} \mathrm{ClO}^{\circ} / \mathrm{ClO}_{-}=2.35 \pm 0.05 \mathrm{~V} \text {. }
$$

From this we obtain that the standard Gibbs energy of the reaction

$$
\mathrm{ClO}_{3} \cdot(\text { aq })+1 / 2 \mathrm{H}_{2} \text { (gas) } \rightarrow \mathrm{ClO}_{3}{ }^{-} \text {(aq) }+\mathrm{H}^{+} \text {(gas) }
$$

is $\Delta_{\mathrm{S} 3} G^{\circ}=-\mathrm{FE}^{\circ} \mathrm{ClO3}^{\circ} / \mathrm{ClO}_{-}=-227 \pm 5 \mathrm{~kJ} / \mathrm{mol}$. Value (S2) corresponds to a temperature of $20{ }^{\circ} \mathrm{C}=293 \mathrm{~K}$; we assume that at $298 \mathrm{~K}$ the value of $E^{\circ} \mathrm{ClO}^{\circ} / \mathrm{ClO}_{3}-$ does not significantly differ from (S2). Then we can take $-227 \pm 5 \mathrm{~kJ} / \mathrm{mol}$ as an estimate of the standard Gibbs energy of reaction (S3) at $298 \mathrm{~K}$,

$$
\Delta_{\mathrm{S} 3} G^{\circ}{ }_{298}=-227 \pm 5 \mathrm{~kJ} / \mathrm{mol} \text {. }
$$

Taking into account $\Delta_{f} G^{\circ}{ }_{298}\left(\mathrm{ClO}_{3}{ }^{-}\right.$,aq $)=-7.95 \mathrm{~kJ} / \mathrm{mol}$ [69], we obtain the Gibbs energy of formation of $\mathrm{ClO}_{3}$ free radical in aqueous solution:

$$
\Delta_{f} G^{\circ}{ }_{298}\left(\mathrm{ClO}_{3}{ }^{\circ}, \mathrm{aq}\right)=\Delta_{f} G^{\circ}{ }_{298}\left(\mathrm{ClO}_{3}{ }^{-}, \mathrm{aq}\right)-\Delta_{\mathrm{S}_{3}} G^{\circ}{ }_{298}=219 \pm 5 \mathrm{~kJ} / \mathrm{mol} \text {. }
$$

Using reference data $\Delta_{f} G^{\circ}{ }_{298}\left(\mathrm{O}_{3}, \mathrm{aq}\right)=174.1 \mathrm{~kJ} / \mathrm{mol}, \Delta_{f} G^{\circ}{ }_{298}\left(\mathrm{ClO}_{2}{ }^{\circ}, \mathrm{aq}\right)=$ $=120.1 \mathrm{~kJ} / \mathrm{mol}, \Delta_{f} G^{\circ}{ }_{298}\left(\mathrm{O}_{2}, \mathrm{aq}\right)=16.4 \mathrm{~kJ} / \mathrm{mol}[69]$ and the result $(\mathrm{S} 4)$, we have for the reaction

$$
\begin{gathered}
\mathrm{ClO}_{3} \cdot(\mathrm{aq})+\mathrm{O}_{3}(\mathrm{aq}) \rightarrow \mathrm{ClO}_{2} \cdot(\mathrm{aq})+2 \mathrm{O}_{2}(\mathrm{aq}) \\
\Delta_{\mathrm{S} 5} G^{\circ}=120.1+2 \cdot 16.4-219 \pm 5-174.1= \\
=-240 \pm 5 \mathrm{~kJ} / \mathrm{mol} .
\end{gathered}
$$




\section{REFERENCES}

1. Wilhelm E., Battino R., Wilcock R.J. Low-pressure solubility of gases in liquid water. Chem. Rev. 1977, 77(2): 219-262.

2. Levanov A.V., Isaikina O.Y., Gasanova R.B., Lunin V.V. Solubility of Ozone and Kinetics of Its Decomposition in Aqueous Chloride Solutions. Ind. Eng. Chem. Res. 2018, 57(43): 14355-14364.

3. Aieta E.M., Roberts P.V. Henry constant of molecular chlorine in aqueous solution. J. Chem. Eng. Data 1986, 31(1): 51-53.

4. Huthwelker T., Peter T., Luo B.P., Clegg S.L., Carslaw K.S., Brimblecombe P. Solubility of $\mathrm{HOCl}$ in water and aqueous H2SO4 to stratospheric temperatures. J Atmos Chem 1995, 21(1): 81-95.

5. Bräuer P., Tilgner A., Wolke R., Herrmann H. Mechanism development and modelling of tropospheric multiphase halogen chemistry: The CAPRAM Halogen Module 2.0 (HM2). Electronic Supplementary Material. J. Atmos. Chem. 2013, 70(1): 19-52.

6. CRC Handbook of Chemistry and Physics, 90th Edition (CD-ROM Version 2010). Boca Raton, FL., USA: CRC Press/Taylor and Francis, 2010.

7. Elliot A.J., Bartels D.M., The Reaction Set, Rate Constants and g-Values for the Simulation of the Radiolysis of Light Water over the Range $20^{\circ}$ to $350^{\circ} \mathrm{C}$ Based on Information Available in 2008. Report AECL No. 153-127160450-001. Mississauga (Ontario, Canada): Atomic Energy of Canada Ltd 2009.

8. Poskrebyshev G.A., Neta P., Huie R.E. Temperature Dependence of the Acid Dissociation Constant of the Hydroxyl Radical. J. Phys. Chem. A 2002, 106(47): 11488-11491.

9. Buehler R.E., Staehelin J., Hoigne J. Ozone Decomposition in Water Studied by Pulse Radiolysis. 1. Perhydroxyl (HO2)/Hyperoxide (O2-) and HO3/O3- as Intermediates. J. Phys. Chem. 1984, 88(12): 2560-2564.

10. Buehler R., Staehelin J., Hoigne J. Ozone Decomposition in Water Studied by Pulse Radiolysis 1. HO2/O2- and HO3/O3- as Intermediates. Correction. J. Phys. Chem. 1984, 88(22): 5450-5450.

11. Morris J.C. The Acid Ionization Constant of HOCl from 5 to $35^{\circ}$. J. Phys. Chem. 1966, 70(12): 3798-3805. 
12. Adam L.C., Fábián I., Suzuki K., Gordon G. Hypochlorous Acid Decomposition in the pH 5-8 Region. Inorg. Chem. 1992, 31(17): 35343541 .

13. Fabian I., Gordon G. Complex formation reactions of the chlorite ion. Inorganic Chemistry 1991, 30(19): 3785-3787.

14. Wang T.X., Margerum D.W. Kinetics of Reversible Chlorine Hydrolysis: Temperature Dependence and General-Acid/Base-Assisted Mechanisms. Inorg. Chem. 1994, 33(6): 1050-1055.

15. Ershov B.G., Janata E., Kelm M., Gordeev A.V. Formation and absorption spectra of X3-ions upon the radiation-chemical oxidation of $\mathrm{Cl}$-in the presence of $\mathrm{Br}-(\mathrm{Cl}, \mathrm{Br}=X)$ in aqueous solution. Mendeleev Comm. 2002, 12(2): 55-57.

16. Wang T.X., Kelley M.D., Cooper J.N., Beckwith R.C., Margerum D.W. Equilibrium, Kinetic, and UV-Spectral Characteristics of Aqueous Bromine Chloride, Bromine, and Chlorine Species Inorg. Chem. 1994, 33(25): 58725878

17. Levanov A.V., Isaikina O.Y., Gasanova R.B., Lunin V.V. Coefficient of Ozone Mass Transfer during Its Interaction with an Aqueous Solution of Formic Acid in a Bubble Column Reactor. Russ. J. Phys. Chem. A 2017, 91(8): 1427-1431.

18. Gershenzon M., Davidovits P., Jayne J.T., Kolb C.E., Worsnop D.R. Rate Constant for the Reaction of Cl2(aq) with $\mathrm{OH}-$. J. Phys. Chem. A 2002, 106(34): 7748-7754.

19. Haag W.R., Hoigné J. Ozonation of Water Containing Chlorine or Chloramines. Reaction Products and Kinetics. Water Res. 1983, 17(10): 1397-1402.

20. Klaning U.K., Sehested K., Holcman J. Standard Gibbs energy of formation of the hydroxyl radical in aqueous solution. Rate constants for the reaction $\mathrm{ClO} 2-+\mathrm{O} 3=\mathrm{O} 3-+\mathrm{ClO} 2$. J. Phys. Chem. 1985, 89(5): 760-763.

21. Nicoson J.S., Wang L., Becker R.H., Huff Hartz K.E., Muller C.E., Margerum D.W. Kinetics and Mechanisms of the Ozone/Bromite and Ozone/Chlorite Reactions. Inorg. Chem. 2002, 41(11): 2975-2980.

22. Zuo Z., Katsumura Y., Ueda K., Ishigure K. Reactions between some inorganic radicals and oxychlorides studied by pulse radiolysis and laser photolysis. J. Chem. Soc. Faraday Trans. 1997, 93(10): 1885-1891. 
23. Eriksen T.E., Lind J., Merenyi G. Generation of chlorine dioxide from ClO2- by pulse radiolysis. J. Chem. Soc. Faraday Trans. 1 1981, 77(9): 2115-2123.

24. Hoigné J., Bader H. Kinetics of reactions of chlorine dioxide (OClO) in water. I. Rate constants for inorganic and organic compounds. Water Res. 1994, 28(1): 45-55.

25. Buxton G.V., Subhani M.S. Radiation chemistry and photochemistry of oxychlorine ions. Part 1. Radiolysis of aqueous solutions of hypochlorite and chlorite ions. J. Chem. Soc. Faraday Trans. 1 1972, 68: 947-957.

26. Klaning U.K., Sehested K. The primary process $\mathrm{ClO}_{-}-+(\mathrm{hv}) \rightarrow \mathrm{ClO}-+$ $\mathrm{O} 2$ in the photolysis of aqueous ClO3-solutions. J. Phys. Chem. 1991, 95(2): 740-743.

27. Yu X.Y. Critical evaluation of rate constants and equilibrium constants of hydrogen peroxide photolysis in acidic aqueous solutions containing chloride ions. Journal of Physical and Chemical Reference Data 2004, 33(3): 747-763.

28. Klaning U.K., Wolff T. Laser Flash Photolysis of $\mathrm{HClO}, \mathrm{ClO}^{-}, \mathrm{HBrO}$, and $\mathrm{BrO}^{-}$in Aqueous Solution. Reactions of $\mathrm{Cl}$ - and Br-Atoms. Ber. Bunsenges. phys. Chem. 1985, 89(3): 243-245.

29. Jayson G.G., Parsons B.J., Swallow A.J. Some Simple, Highly Reactive, Inorganic Chlorine Derivatives in Aqueous-Solution. Their Formation Using Pulses of Radiation and Their Role in Mechanism of Fricke Dosimeter. J. Chem. Soc. Faraday Trans. I 1973, (9): 1597-1607.

30. Grigorev A.E., Makarov I.E., Pikaev A.K. Formation of Cl2- in the Bulk Solution during the Radiolysis of Concentrated Aqueous Solutions of Chlorides. High Energy Chemistry 1987, 21(2): 99-102.

31. Wu D., Wong D., Di Bartolo B. Evolution of $\mathrm{Cl} 2-$ in aqueous $\mathrm{NaCl}$ solutions. J. Photochem. 1980, 14(4): 303-310.

32. Wagner I., Karthäuser J., Strehlow H. On the Decay of the Dichloride Anion Cl2- in Aqueous Solution. Ber. Bunsenges. phys. Chem. 1986, 90(10): 861867.

33. Bielski B.H.J. A Pulse Radiolysis Study of the Reaction of Ozone with Cl2Radical-Anion in Aqueous Solutions. Rad. Phys. Chem. 1993, 41(3): 527530. 
34. Alfassi Z.B., Huie R.E., Mosseri S., Neta P. Kinetics of one-electron oxidation by the ClO radical. Rad. Phys. Chem. (Int. J. Rad. Appl. Instr. Part C) 1988, 32(1): 85-88.

35. Mialocq J.C., Barat F., Gilles L., Hickel B., Lesigne B. Flash photolysis of chlorine dioxide in aqueous solution. J. Phys. Chem. 1973, 77(6): 742-749.

36. Klaning U.K., Sehested K., Wolff T. Ozone formation in laser flash photolysis of oxoacids and oxoanions of chlorine and bromine. J. Chem. Soc. Faraday Trans. 1 1984, 80(11): 2969-2979.

37. Mohammad A., Liebhafsky H.A. The Kinetics of the Reduction of Hydrogen Peroxide by the Halides. J. Am. Chem. Soc. 1934, 56(8): 1680-1685.

38. Held A.M., Halko D.J., Hurst J.K. Mechanisms of Chlorine Oxidation of Hydrogen Peroxide. J. Am. Chem. Soc. 1978, 100(18): 5732-5740.

39. Makower B., Bray W.C. The Rate of Oxidation of Hydrogen Peroxide by Chlorine in the Presence of Hydrochloric Acid. Journal of the American Chemical Society 1933, 55(12): 4765-4776.

40. Connick R.E. The Interaction of Hydrogen Peroxide and Hypochlorous Acid in Acidic Solutions Containing Chloride Ion. Journal of the American Chemical Society 1947, 69(6): 1509-1514.

41. Ruiz-Ibanez G., Sandall O.C. Kinetics for the reaction between chlorine and basic hydrogen peroxide. Ind. Eng. Chem. Res. 1991, 30(6): 1105-1110.

42. Davis R.A., Ruiz-Ibanez G., Sandall O.C. Nonisothermal analysis of the reaction kinetics for chlorine in basic hydrogen peroxide. Ind. Eng. Chem. Res. 1992, 31(6): 1461-1465.

43. Bjergbakke E., Navaratnam S., Parsons B.J., Swallow A.J. Reaction between HO2 and Chlorine in Aqueous Solution. J. Am. Chem. Soc. 1981, 103(19): 5926-5928.

44. Long C.A., Bielski B.H.J. Rate of reaction of superoxide radical with chloride-containing species. J. Phys. Chem. 1980, 84(5): 555-557.

45. Graedel T.E., Goldberg K.I. Kinetic Studies of Raindrop Chemistry. 1. Inorganic and Organic Processes. J. Geophys. Res. Oceans Atmospheres 1983, 88(NC15): 865-882.

46. Zhestkova T.P., Pikaev A.K. Destruction rate of Cl2-anion-radicals during pulse radiolysis of concentrated aqueous lithium chloride solutions. Bull. Acad. Sci. USSR. Div. chem. sci. 1974, 23(4): 877-878. 
47. Huie R.E., Neta P. Kinetics of one-electron transfer reactions involving chlorine dioxide and nitrogen dioxide. J. Phys. Chem. 1986, 90(6): 11931198.

48. Quiroga S.L., Perissinotti L.J. Reduced mechanism for the $366 \mathrm{~nm}$ chlorine dioxide photodecomposition in N2-saturated aqueous solutions. J.

Photochem. Photobiol. A: Chemistry 2005, 171(1): 59-67.

49. Staehelin J., Hoigne J. Decomposition of ozone in water: rate of initiation by hydroxide ions and hydrogen peroxide. Env. Sci. Technol. 1982, 16(10): 676-681.

50. Sehested K., Corfitzen H., Holcman J., Hart E.J. Decomposition of ozone in aqueous acetic acid solutions (pH 0-4). J. Phys. Chem. 1992, 96(2): 10051009.

51. Sehested K., Holcman J., Bjergbakke E., Hart E.J. A pulse radiolytic study of the reaction $\mathrm{OH}+\mathrm{O} 3$ in aqueous medium. J. Phys. Chem. 1984, 88(18): 4144-4147.

52. Gonzalez M.C., Mártire D.O. Kinetics of $\mathrm{O}^{\cdot-}$ and $\mathrm{O3} \cdot-$ in alkaline aqueous solutions of hydrogen peroxide. Int. J. Chem. Kin. 1997, 29(8): 589-597.

53. Sehested K., Holcman J., Hart E.J. Rate constants and products of the reactions of $e_{a q}-\mathrm{O}_{2}-$ and $\mathrm{H}$ with ozone in aqueous solutions. J. Phys. Chem. 1983, 87(11): 1951-1954.

54. Yu X.-Y., Barker J.R. Hydrogen Peroxide Photolysis in Acidic Aqueous Solutions Containing Chloride Ions. I. Chemical Mechanism. J. Phys. Chem. A 2003, 107(9): 1313-1324.

55. Sehested K., Holcman J., Bjergbakke E., Hart E.J. Use of pulse radiolysis for the study of the chemistry of aqueous ozone and ozonide solutions. J. Radioanalyt. Nucl. Chem. 1986, 101(2): 239-250.

56. Staehelin J., Buehler R.E., Hoigne J. Ozone decomposition in water studied by pulse radiolysis. 2. Hydroxyl and hydrogen tetroxide (HO4) as chain intermediates. J. Phys. Chem. 1984, 88(24): 5999-6004.

57. Sehested K., Holcman J., Bjergbakke E., Hart E.J. Formation of ozone in the reaction of hydroxyl with $\mathrm{O3}$ - and the decay of the ozonide ion radical at pH 10-13. J. Phys. Chem. 1984, 88(2): 269-273.

58. Kester D.R., Pytkowicx R.M. Determination of the Apparent Dissociation Constants of Phosphoric Acid in Seawater. Limnol. Oceanogr. 1967, 12(2): 243-252. 
59. Adam L.C., Fábián I., Suzuki K., Gordon G. Hypochlorous Acid Decomposition in the pH 5-8 Region. Inorganic Chemistry 1992, 31(17): 3534-3541.

60. Nemes A., Fábián I., Gordon G. The Kinetics and Mechanism of Aqueous Ozone Decomposition in Alkaline Solution. Inorg. React. Mech. 2000, 2: 327-342.

61. Tomiyasu H., Fukutomi H., Gordon G. Kinetics and mechanism of ozone decomposition in basic aqueous solution. Inorg. Chem. 1985, 24(19): 29622966.

62. Bezbarua B.K., Reckhow D.A. Modification of the Standard Neutral Ozone Decomposition Model. Ozone Sci. Eng. 2004, 26(4): 345-357.

63. Nemes A., Fábián I., Van Eldik R. Kinetics and Mechanism of the Carbonate Ion Inhibited Aqueous Ozone Decomposition. J. Phys. Chem. A 2000, 104(34): 7995-8000.

64. Elovitz M.S., Von Gunten U. Hydroxyl Radical/Ozone Ratios During Ozonation Processes. I. The Rct Concept. Ozone Sci. Eng. 1999, 21(3): 239260.

65. Levanov A.V., Isaikina O.Y., Gasanova R.B., Uzhel A.S., Lunin V.V. Kinetics of chlorate formation during ozonation of aqueous chloride solutions. Chemosphere 2019, 229: 68-76.

66. Kang N., Jackson W.A., Dasgupta P.K., Anderson T.A. Perchlorate production by ozone oxidation of chloride in aqueous and dry systems. Sci. Total Environ. 2008, 405(1-3): 301-309.

67. Rao B., Anderson T.A., Redder A., Jackson W.A. Perchlorate Formation by Ozone Oxidation of Aqueous Chlorine / Oxy-Chlorine Species : Role of ClxOy Radicals. Env. Sci. Technol. 2010, 44(8): 2961-2967.

68. Zuo Z., Katsumura Y., Ueda K., Ishigure K. Laser photolysis study on reactions of sulfate radical and nitrate radical with chlorate ion in aqueous solutions Formation and reduction potential of $\mathrm{ClO} 3$ radical. J. Chem. Soc. Faraday Trans. 1997, 93(4): 533-536.

69. Wagman D.D., Evans W.H., Parker V.B., Schumm R.H., Halow I., Bailey S.M., Churney K.L., Nutall R.L., The NBS Tables of Chemical Thermodynamic Properties. J. Phys. Chem. Ref. Data, 1982, 11, Supplement №2. Washington D.C.: National Bureau of Standards, 1982. 